\title{
Banach spaces adapted to Anosov systems
}

\author{
SÉBASTIEN GOUËZEL $\dagger$ and CARLANGELO LIVERANI† \\ $\dagger$ IRMAR, Université de Rennes 1, Campus de Beaulieu, bâtiment 22, \\ 35042 Rennes Cedex, France \\ (e-mail: sebastien.gouezel@univ-rennes1.fr) \\ $\ddagger$ Dipartimento di Matematica, II Università di Roma (Tor Vergata), \\ Via della Ricerca Scientifica, 00133 Roma, Italy \\ (e-mail: liverani@mat.uniroma2.it)
}

(Received 8 March 2005 and accepted in revised form 31 May 2005)

\begin{abstract}
We study the spectral properties of the Ruelle-Perron-Frobenius operator associated to an Anosov map on classes of functions with high smoothness. To this end we construct anisotropic Banach spaces of distributions on which the transfer operator has a small essential spectrum. In the $\mathcal{C}^{\infty}$ case, the essential spectral radius is arbitrarily small, which yields a description of the correlations with arbitrary precision. Moreover, we obtain sharp spectral stability results for deterministic and random perturbations. In particular, we obtain differentiability results for spectral data (which imply differentiability of the Sinai-Ruelle-Bowen measure, the variance for the central limit theorem, the rates of decay for smooth observable, etc.).
\end{abstract}

\section{Introduction}

The study of the statistical properties of Anosov systems dates back almost half a century [1] and many approaches have been developed to investigate various aspects of the field (the most historically relevant one being based on the introduction of Markov partitions $[\mathbf{2}, \mathbf{8}, \mathbf{2 4}, \mathbf{3 1}])$. At the same time the types of question and the precision of the results have progressed over the years. In the last few years the emphasis has been on strong stability properties with respect to various types of perturbation [4], dynamical zeta functions and the related smoothness issue (see $[\mathbf{9 , 1 4 , 2 6 ] ) . ~ I n ~ t h e ~ p r e s e n t ~ p a p e r ~ w e ~}$ present a new approach, improving on the previous partial and still unsatisfactory method by Blank et al that allows one to obtain easily an array of results (many of which are new) and we hope will reveal an even larger field of applicability. Indeed, the ideas in [7] have already been applied with success to some partially hyperbolic situations (flows) [19] and we expect them to be applicable to the study of dynamical zeta functions.

The basic idea is inspired by the work on piecewise expanding maps, starting with $[12,15]$ and the many others that contributed subsequently (see [4] for a review 
on the subject). This means studying directly the transfer operator (often called the Ruelle-Perron-Frobenius operator) on appropriate functional spaces $\dagger$. For the case of smooth expanding maps, the Sobolev spaces $W^{r, 1}$, or the Banach spaces $\mathcal{C}^{r}$, turn out to be proper spaces where the transfer operator acts as a smoothing operator [25] (see [17] for a pedestrian introduction) $\ddagger$. In turn, this implies that, on such spaces, the operator is quasicompact with an essential spectral radius exponentially decreasing in $r$. The existence of a spectral gap and all kind of statistical properties (exponential decay of correlations, central limit theorem, meromorphic zeta functions, etc.) readily follow.

Unfortunately, for Anosov systems it is not helpful to consider spaces of smooth functions - on such spaces the spectral radius of the transfer operator is larger than oneit is necessary to consider spaces of distributions. This was recognized in $[\mathbf{1 0 , 2 8 - 3 0}]$ limited to the analytic case, and in [16] (only implicitly) and systematically in [7] for the $\mathcal{C}^{1+\alpha}$ case. Nevertheless, the latter setting still had several shortcomings. First, the Banach space was precisely patterned on the invariant distributions of the systems, which implied that transfer operators - even of close maps-were studied on different spaces. This was a serious obstacle to obtaining sharp perturbation results. Second as in general the invariant distributions are only Hölder, it was not possible to have a scale of Banach spaces on which to study the influence of the smoothness of the map on the spectrum.

Both such shortcomings are overcome in the present approach. The spaces we introduce (partially inspired by [16]) are still related to the map one wishes to study, but in a much loser way so that the operators associated to nearby maps can be studied on the same space. In addition, we have a scale of spaces that can be used to investigate smoothness related issues (typically the dependence of the essential spectrum on the smoothness of the map). In particular, if the map is $\mathcal{C}^{\infty}$, we obtain a description of the correlations of $\mathcal{C}^{\infty}$ functions with an arbitrarily small error term.

In addition, the present norms allow easier estimates of the size of perturbations. This provides a very direct way of obtaining sharp perturbations results which substantially generalize the existing ones, e.g. $[3,7,23,26,27]$.

For example, in the $\mathcal{C}^{\infty}$ case all the simple eigenvalues and all the eigenspaces depend $\mathcal{C}^{\infty}$ on the map. The same holds for the variance in the central limit theorem (CLT) for a smooth zero average observable.

A further remarkable feature of the present approach is that, unlike all the previous ones (excepted [3]), its implementation does not depend directly on subtle regularity properties of the foliations and of the holonomies. This makes it possible to have a much simpler and self-contained treatment of the statistical properties of the system and may lead to interesting generalizations in the partially hyperbolic setting.

The paper is organized as follows. In $\S 2$, we introduce Banach spaces $\mathcal{B}^{p, q}$, explain why the transfer operator acting on $\mathcal{B}^{p, q}$ has a spectral gap and illustrate the stability

\footnotetext{
$\dagger$ Lasota and Yorke [15], to our knowledge, have given the first Markov-partition-free approach to the study of the invariant measure of systems with sensitive dependence on initial conditions, while Keller [12] has given the first Markov-partition-free approach for the study of general statistical properties. Such papers where limited to expanding systems; for hyperbolic systems Markov-partition-free approaches to the study of the invariant measures where first developed in $[\mathbf{2 0}, \mathbf{2 2}]$.

\$ The choice of $\mathcal{C}^{r}$, which requires a little more work (analogous to the argument at the end of $\$ 6.1$ here) is the choice generalized by the spaces we introduce in this paper.
} 
results: the main ingredients are a compactness statement (Lemma 2.1), a Lasota-Yorke type inequality (Lemma 2.2) and the estimates on perturbations Lemmas 7.1 and 7.2. In $\S \S 3$ and 4 , we describe more precisely the spaces $\mathcal{B}^{p, q}$ and prove in particular that they are spaces of distributions. In $\$ \S 5$ and 6 , which are the main parts of this article, we prove respectively the aforementioned compactness statement and Lasota-Yorke-type inequality. In $\S 7$, we show how this framework implies very precise stability results on the spectrum, for deterministic and random perturbations. Section 8 contains an abstract perturbation result generalizing the setting of [13], along the direction adumbrated in [17], to cases where a control on the smoothness is available. Section 9 shows that smooth deterministic perturbations fit in the setting developed in $\S 8$. Finally, an appendix contains the proof of an intuitive, but technical, result.

Remark 1.1. After the preprint version of this paper was made available, Baladi and Tsujii [6] introduced a quite different approach, using Fourier transform techniques, which makes it possible to remove the only limitation in our approach, that is, the fact that the spectral parameter $p$ has to be an integer (see Remark 2.4).

\section{The Banach spaces and the results}

For $q \geq 0$, let $\lfloor q\rfloor$ be its integer part. We denote by $\overline{\mathcal{C}}^{q}$ the set of functions which are $\lfloor q\rfloor$ times continuously differentiable, and whose $\lfloor q\rfloor$ th derivative is Hölder continuous of exponent $q-\lfloor q\rfloor$ if $q$ is not an integer. To fix the notation, in this paper we choose, for each $q \in \mathbb{R}_{+}$, a norm on $\overline{\mathcal{C}}^{q}$ functions so that $\left|\varphi_{1} \varphi_{2}\right|_{\mathcal{C}^{q}} \leq\left|\varphi_{1}\right|_{\mathcal{C}^{q}}\left|\varphi_{2}\right|_{\mathcal{C}^{q}}$. We will denote by $\mathcal{C}^{q}$ the closure in $\overline{\mathcal{C}}^{q}$ of the set of $\mathcal{C}^{\infty}$ functions. It coincides with $\overline{\mathcal{C}}^{q}$ if $q$ is an integer, but is strictly included in it otherwise. In any case, it contains $\overline{\mathcal{C}} q^{\prime}$ for all $q^{\prime}>q$.

Let $X$ be a $d$-dimensional $\mathcal{C}^{\infty}$ compact connected Riemannian manifold and consider an Anosov map $T \in \mathcal{C}^{r+1}(X, X)$ (for some real $r>1$ ). Write $d_{s}$ and $d_{u}$ for the stable and unstable dimensions. Let $\lambda>1$ be less than the minimal expansion along the unstable directions, and $v<1$ be greater than the minimal contraction along the stable directions. We will express the spectral properties of $T$ using the constants $\lambda$ and $\nu$.

In $\S 3$, we will define a set $\Sigma$ of admissible leaves. The elements of $\Sigma$ are small $\mathcal{C}^{r+1}$ embedded compact manifolds with boundary, of dimension $d_{s}$, close to local stable manifolds $\dagger$.

In what follows, if $v$ is a smooth vector field on an open subset of $X$ and $f$ is a smooth function, then $v f$ will denote the derivative of $f$ in the direction $v$. If $v_{1}, \ldots, v_{p}$ are smooth vector fields, then $v_{1} \cdots v_{p} f$ will denote $v_{1}\left(v_{2}\left(\cdots\left(v_{p} f\right)\right) \cdots\right)$. We will sometimes write $\prod_{i=1}^{p} v_{i} f$ for this expression, although it may be a little misleading as the vector fields $v_{i}$ do not necessarily commute.

We are now ready to introduce the relevant norms. When $W \in \Sigma$, we will denote by $\mathcal{C}_{0}^{q}(W, \mathbb{R})$ the set of functions from $W$ to $\mathbb{R}$ which belong to $\mathcal{C}^{q}$ and vanish on a neighborhood of the boundary of $W$, and by $\mathcal{V}^{r}(W)$ the set of $\mathcal{C}^{r}$ vector fields defined on a neighborhood of $W$.

$\dagger$ The precise definition of the set $\Sigma$ is given by (3.2). 
For each $h \in \mathcal{C}^{r}(X, \mathbb{R})$ and $q \in \mathbb{R}_{+}, p \in \mathbb{N}$ with $p \leq r$ (recall, $T$ is $\mathcal{C}^{r+1}$ by definition), let $\dagger$

$$
\|h\|_{p, q}^{-}:=\sup _{W \in \Sigma} \sup _{\substack{v_{1}, \ldots, v_{p} \in \mathcal{V}^{r}(W) \\\left|v_{i}\right|_{\mathcal{C}^{r} \leq 1}}} \sup _{\substack{\varphi \in \mathcal{C}_{0}^{q}(W, \mathbb{R}) \\|\varphi|_{\mathcal{C}^{q} \leq 1}}} \int_{W} v_{1} \cdots v_{p} h \cdot \varphi .
$$

It satisfies $\|h\|_{p, q^{\prime}}^{-} \leq\|h\|_{p, q}^{-}$if $q^{\prime} \geq q$. Then define the norms

$$
\|h\|_{p, q}=\sup _{0 \leq k \leq p}\|h\|_{k, q+k}^{-}=\sup _{p^{\prime} \leq p, q^{\prime} \geq q+p^{\prime}}\|h\|_{p^{\prime}, q^{\prime}}^{-} .
$$

For example, if $X$ is the torus, the above norm is equivalent to the one given by

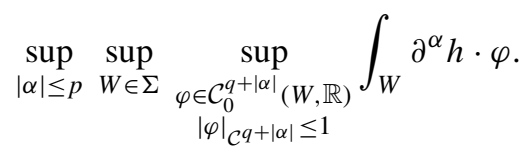

Later on, in $\S 3$, we will give an explicit description of the norm (2.2) in coordinate charts. It will sometimes be easier to work with the coordinate-free definition given in (2.2) and sometimes with the explicit definition, depending on what we are trying to prove.

It is easy to see that $\|\cdot\|_{p, q}$, for $p \leq r$, is a norm on $\mathcal{C}^{r}(X, \mathbb{R})$ (we will prove a more general result in Proposition 4.1). Hence, we can consider the completion $\mathcal{B}^{p, q}$ of $\mathcal{C}^{r}(X, \mathbb{R})$ with respect to this norm. Section 4 will be devoted to a description of this space. We will see in particular that it is canonically a space of distributions. More precisely, for $q \leq r$, denote by $\mathcal{D}_{q}^{\prime}$ the dual of the space $\mathcal{C}^{q}(X, \mathbb{R})$. It is the space of distributions of order at most $q$, endowed with its canonical norm. Proposition 4.1 proves that the embedding of $\mathcal{C}^{r}(X, \mathbb{R})$ into $\mathcal{D}_{q}^{\prime}$ extends to a continuous embedding of $\mathcal{B}^{p, q}$ into $\mathcal{D}_{q}^{\prime}$.

As $\|h\|_{p-1, q+1} \leq\|h\|_{p, q}$, the embedding of $\mathcal{C}^{r}(X, \mathbb{R})$ into $\mathcal{B}^{p, q}$ gives rise to a canonical map $\mathcal{B}^{p, q} \rightarrow \mathcal{B}^{p-1, q+1}$, which is in fact compact.

LEMMA 2.1. If $p+q<r$, the unit ball of $\mathcal{B}^{p, q}$ is relatively compact in $\mathcal{B}^{p-1, q+1}$.

The proof of Lemma 2.1 is the content of $\S 5$.

The rest of the paper consists of the investigation of the properties of the transfer operator $\mathcal{L}$ seen as an operator acting on the spaces $\mathcal{B}^{p, q}$. The transfer operator acts as follows on distributions of order at most $r$ : if $h \in \mathcal{D}_{r}^{\prime}$ and $\varphi$ is a $\mathcal{C}^{r}$ test function, then $\mathcal{L} h$ is defined by $\langle\mathcal{L} h, \varphi\rangle=\langle h, \varphi \circ T\rangle$. If $h$ is a continuous function, then the distribution $\mathcal{L} h$ is in fact still a function, and is given by the formula

$$
\mathcal{L} h=\left(h|\operatorname{det}(D T)|^{-1}\right) \circ T^{-1} .
$$

As $\mathcal{B}^{p, q}$ is contained in $\mathcal{D}_{r}^{\prime}$, the transfer operator acts on elements of $\mathcal{B}^{p, q}$, and their images still belong to $\mathcal{B}^{p, q}$. The key information on the action of $\mathcal{L}$ on $\mathcal{B}^{p, q}$ is contained in the next lemma.

LEMMA 2.2. For each $p \in \mathbb{N}$ and $q \geq 0$ satisfying $p+q<r$, $\mathcal{L}$ is a bounded operator on $\mathcal{B}^{p, q}$. In addition, there exist $A_{p, q}, B_{p, q}>0$ such that, for each $n \in \mathbb{N}$,

$$
\begin{gathered}
\left\|\mathcal{L}^{n} h\right\|_{0, q} \leq A_{0, q}\|h\|_{0, q}, \\
\left\|\mathcal{L}^{n} h\right\|_{p, q} \leq A_{p, q} \max \left(\lambda^{-p}, v^{q}\right)^{n}\|h\|_{p, q}+B_{p, q}\|h\|_{p-1, q+1} .
\end{gathered}
$$

$\dagger$ All integrals are taken with respect to Lebesgue or Riemannian measure except when another measure is explicitly mentioned. 
The above lemma is proven in $\S 6$.

Lemmas 2.1 and 2.2 readily imply the basic result of the paper.

THEOREM 2.3. If $p \in \mathbb{N}^{*}$ and $q \in \mathbb{R}_{+}^{*}$ satisfy $p+q<r$, then the operator $\mathcal{L}: \mathcal{B}^{p, q} \rightarrow$ $\mathcal{B}^{p, q}$ has spectral radius one. In addition, $\mathcal{L}$ is quasi-compact with essential spectrum $\sigma_{\text {ess }}(\mathcal{L}) \subset\left\{z \in \mathbb{C}:|z| \leq \max \left(\lambda^{-p}, v^{q}\right)\right\}$.

Moreover, the eigenfunctions corresponding to eigenvalues of modulus one are distributions of order zero, i.e. measures. If the map is topologically transitive, then one is a simple eigenvalue, the corresponding eigenfunction is the Sinai-Ruelle-Bowen (SRB) measure, and no other eigenvalue of modulus one is present.

Proof. The first assertion follows from (2.4) as $\|h\|_{p-1, q+1} \leq\|h\|_{p, q}$. The proof of the second is completely standard and can be based, for example, on an argument by Hennion after a spectral formula due to Nussbaum (see [7, Theorem 1] for details).

As the operator $\mathcal{L}: \mathcal{B}^{p, q} \rightarrow \mathcal{B}^{p, q}$ is quasi-compact, it can be written as $\mathcal{L}=$ $\sum_{j=1}^{N} e^{i \theta_{j}} \Pi_{j}+R$, where $\theta_{j} \in \mathbb{R}, \Pi_{j}: \mathcal{B}^{p, q} \rightarrow \mathcal{B}^{p, q}$ is a projection on a finite-dimensional subspace, $\Pi_{j} \Pi_{l}=\delta_{i l} \Pi_{j}, \Pi_{j} R=R \Pi_{j}=0$ and $\left\|R^{n}\right\|_{p, q} \leq C \sigma^{n}$ for some $\sigma<1 \dagger$. This readily implies that

$$
\lim _{n \rightarrow \infty} \frac{1}{n} \sum_{k=0}^{n-1} e^{-i k \theta} \mathcal{L}^{k}= \begin{cases}\Pi_{j} & \text { if } \theta=\theta_{j} \\ 0 & \text { otherwise. }\end{cases}
$$

In addition, for each $h, \varphi \in \mathcal{C}^{r}$,

$$
\left|\left\langle\Pi_{j} h, \varphi\right\rangle\right|=\lim _{n \rightarrow \infty} \frac{1}{n}\left|\sum_{k=0}^{n-1} e^{-i k \theta_{j}}\left\langle h, \varphi \circ T^{k}\right\rangle\right| \leq|h|_{\mathcal{C}^{0}}|\varphi|_{\mathcal{C}^{0}} .
$$

The subspace $\Pi_{j} \mathcal{C}^{r}$ is dense in the finite-dimensional space $\Pi_{j} \mathcal{B}^{p, q}$, therefore $\Pi_{j} \mathcal{C}^{r}=$ $\Pi_{j} \mathcal{B}^{p, q}$. Hence, the above means exactly that the range of the projectors (that is the eigenvectors) are measures. The rest of the theorem is then a consequence of the ergodic decomposition.

Remark 2.4. If $\lambda^{-1}=v$ and $r$ is an even integer, then the optimal choice of $p, q$ in Theorem 2.3 is $p=r / 2$ and $q=r / 2-\varepsilon$ for some arbitrarily small $\varepsilon>0$. Such a condition is similar to Kitaev's requirement [14] that, in our language, reads $p=q=r / 2$. Hence, our results are probably optimal in this case. However, in the general case, our results are limited by the fact that $p$ has to be an integer. It would probably require significantly different and less elementary techniques to allow $p \in \mathbb{R}_{+}$as this would require the definition of 'fractional derivatives'. See [5] for a very recent attempt, based on Fourier analysis, in a special case, and [6] for an even more recent and completely general approach.

Remark 2.5. The space $\mathcal{B}^{p, q}$ depends on the choice of the set $\Sigma$ of admissible leaves. However, choosing a different $\widetilde{\Sigma}$ and $\widetilde{\mathcal{B}}^{p, q}$ would not change the spectral properties of $\mathcal{L}$. Namely, in this case, there exists $n \in \mathbb{N}$ such that $\mathcal{L}^{n}\left(\mathcal{B}^{p, q}\right) \subset \widetilde{\mathcal{B}}^{p, q}$ and $\mathcal{L}^{n}\left(\widetilde{\mathcal{B}}^{p, q}\right) \subset \mathcal{B}^{p, q}$, whence the spectral data of $\mathcal{L}$ acting on $\mathcal{B}^{p, q}$ or $\widetilde{\mathcal{B}}^{p, q}$ are the same.

$\dagger$ The reason why no Jordan blocks can be associated to the modulus one eigenvalues is that the $\mathcal{L}^{n}$ are equibounded while a Jordan block would force a polynomial growth. 
In the $\mathcal{C}^{\infty}$ case, Theorem 2.3 immediately implies the following description of the correlations of $\mathcal{C}^{\infty}$ functions.

COROLlary 2.6. Assume that $T$ is $\mathcal{C}^{\infty}$. Then there exist a sequence of complex numbers $\lambda_{k}$ such that $\left|\lambda_{k}\right|$ decreases to zero, and non-negative integers $r_{k}$ such that, for any $f, g: X \rightarrow \mathbb{R}$ of class $\mathcal{C}^{\infty}$, there exist numbers $a_{k}(f, g)$ with

$$
\int f \cdot g \circ T^{n} \sim \sum_{k=0}^{\infty} a_{k}(f, g) n^{r_{k}} \lambda_{k}^{n}
$$

in the following sense. For any $\varepsilon>0$, let $K$ be such that $\left|\lambda_{K}\right|<\varepsilon$. Then

$$
\int f \cdot g \circ T^{n}=\sum_{k=0}^{K-1} a_{k}(f, g) n^{r_{k}} \lambda_{k}^{n}+o\left(\varepsilon^{n}\right) .
$$

The second part of this paper focuses on the spectral stability for a wide class of deterministic and random perturbations. Let us fix $p \in \mathbb{N}^{*}$ and $q>0$ such that $p+q<r$.

Let $U$ be a small enough neighborhood of $T$ in the $\mathcal{C}^{r+1}$ topology. Consider a probability measure $\mu$ on a probability space $\Omega$ and, for $\omega \in \Omega$, take $T_{\omega} \in U$ and $g(\omega, \cdot) \in \mathcal{C}^{p+q}\left(X, \mathbb{R}_{+}\right)$. Assume also that, for all $x \in X, \int g(\omega, x) d \mu(\omega)=1$, and that $\int|g(\omega, \cdot)|_{\mathcal{C}^{p+q}(X, \mathbb{R})} d \mu(\omega)<\infty$. It is then possible to define a random walk in the following way. Starting from a point $x$, choose a diffeomorphism $T_{\omega}$ randomly with respect to the measure $g(\omega, x) d \mu(\omega)$, and go to $T_{\omega}(x)$. Then iterate this process independently.

When $\Omega$ is a singleton and $g(\omega, x)=1$, then this is a deterministic perturbation $T_{\omega}$ of $T$. Random perturbations of the type discussed in [7] can also be described in this way $\dagger$. Hence, this setting encompasses at the same time very general deterministic and random perturbations of $T$. We define the size of the perturbation by

$$
\begin{aligned}
\Delta(\mu, g):= & \int_{\Omega}|g(\omega, \cdot)|_{\mathcal{C}^{p+q}(X, \mathbb{R})} d_{\mathcal{C}^{r+1}}\left(T_{\omega}, T\right) d \mu(\omega) \\
& +\left|x \mapsto \int_{\Omega} g\left(\omega, T_{\omega}^{-1} x\right) d \mu(\omega)-1\right|_{\mathcal{C}^{p+q}(X, \mathbb{R})} .
\end{aligned}
$$

For definiteness, we will fix a large constant $A$ and assume that, until the end of this section, all the perturbations we consider satisfy $\int|g(\omega, \cdot)|_{\mathcal{C}^{p+q}(X, \mathbb{R})} \leq A$.

The transfer operator $\mathcal{L}_{\mu, g}$ associated to the previous random walk is given on $\mathcal{D}_{p+q}^{\prime}$ by $\left\langle\mathcal{L}_{\mu, g} h, \varphi\right\rangle=\langle h, \widetilde{\varphi}\rangle$ where $\tilde{\varphi}(x)=\int_{\Omega} g(\omega, x) \varphi\left(T_{\omega} x\right) d \mu(\omega)$. When $h$ is a function, $\mathcal{L}_{\mu, g} h$ is still a function, given by the following formula:

$$
\mathcal{L}_{\mu, g} h(x)=\int_{\Omega} g\left(\omega, T_{\omega}^{-1}(x)\right) \mathcal{L}_{T_{\omega}} h(x) d \mu(\omega),
$$

where $\mathcal{L}_{T_{\omega}}$ is the transfer operator associated to $T_{\omega}$.

In Lemma 7.1 we show that $\mathcal{L}_{T}$ and $\mathcal{L}_{\widetilde{T}}$ are $\|\cdot\|_{\mathcal{B} p, q \rightarrow \mathcal{B}^{p-1, q+1}}$ close if $T$ and $\tilde{T}$ are close in the $\mathcal{C}^{r+1}$ topology. In turn, this implies that $\mathcal{L}_{T}$ and $\mathcal{L}_{\mu, g}$ are close if $\Delta(\mu, g)$ is small, see (7.4). In addition, it is possible to show that the operators $\mathcal{L}_{\mu, g}$ satisfy a uniform Lasota-Yorke-type inequality (Lemma 7.2). These facts suffice to apply [13] to $\dagger$ To obtain the latter case set $\Omega=\mathbb{T}^{d}, \mu$ is Lebesgue, $T_{\omega}(x)=T x+\omega \bmod 1$ and $g(\omega, x)=q_{\varepsilon}(\omega, T x)$. 
the present context, yielding immediately the strong perturbation results described below greatly generalizing the results in [7].

Fix any $\varrho \in\left(\max \left(\lambda^{-p}, \nu^{q}\right), 1\right)$ and denote by $\operatorname{sp}(\mathcal{L})$ the spectrum of $\mathcal{L}: \mathcal{B}^{p, q} \rightarrow \mathcal{B}^{p, q}$. As the essential spectral radius of $\mathcal{L}$ does not exceed $\max \left(\lambda^{-p}, v^{q}\right)$, the $\operatorname{set} \operatorname{sp}(\mathcal{L}) \cap\{z \in$ $\mathbb{C}:|z| \geq \varrho\}$ consists of a finite number of eigenvalues $\lambda_{1}, \ldots, \lambda_{k}$ of finite multiplicity. Changing $\varrho$ slightly we may assume that $\operatorname{sp}(\mathcal{L}) \cap\{z \in \mathbb{C}:|z|=\varrho\}=\emptyset$. Hence there exists $\delta_{*}<\varrho-\max \left(\lambda^{-p}, \nu^{q}\right)$ such that

$$
\begin{gathered}
\left|\lambda_{i}-\lambda_{j}\right|>\delta_{*} \quad(i \neq j) ; \\
\operatorname{dist}(\operatorname{sp}(\mathcal{L}),\{|z|=\varrho\})>\delta_{*} .
\end{gathered}
$$

THEOREM 2.7. For each $\delta \in\left(0, \delta_{*}\right]$ and $\eta<1-\left(\log \varrho / \log \max \left(\lambda^{-p}, \nu^{q}\right)\right)$, there exists $\varepsilon_{0}$ such that for any perturbation $(\mu, g)$ of $T$ satisfying $\Delta(\mu, g) \leq \varepsilon_{0}$ :

(a) the spectral projectors

$$
\begin{aligned}
\Pi_{\mu, g}^{(j)} & :=\frac{1}{2 \pi \imath} \int_{\left\{\left|z-\lambda_{j}\right|=\delta\right\}}\left(z-\mathcal{L}_{\mu, g}\right)^{-1} d z \\
\Pi_{\mu, g}^{(\varrho)} & :=\frac{1}{2 \pi \imath} \int_{\{|z|=\varrho\}}\left(z-\mathcal{L}_{\mu, g}\right)^{-1} d z
\end{aligned}
$$

are well defined on $\mathcal{B}^{p, q}$; we will denote by $\Pi_{0}^{(j)}$ and $\Pi_{0}^{(\varrho)}$ the corresponding projectors for the unperturbed transfer operator $\mathcal{L}$;

(b) there exists $K_{1}>0$ such that $\left\|\Pi_{\mu, g}^{(j)}-\Pi_{0}^{(j)}\right\|_{\mathcal{B}^{p, q} \rightarrow \mathcal{B}^{p-1, q+1}} \leq K_{1} \Delta(\mu, g)^{\eta}$ and $\left\|\Pi_{\mu, g}^{(\varrho)}-\Pi_{0}^{(\varrho)}\right\|_{\mathcal{B}^{p, q} \rightarrow \mathcal{B}^{p-1, q+1}} \leq K_{1} \Delta(\mu, g)^{\eta}$;

(c) $\operatorname{rank}\left(\Pi_{\mu, g}^{(j)}\right)=\operatorname{rank}\left(\Pi_{0}^{(j)}\right)$;

(d) there exists $K_{2}>0$ such that $\left\|\mathcal{L}_{\mu, g}^{n} \Pi_{\mu, g}^{(\varrho)}\right\|_{\mathcal{B}^{p, q}} \leq K_{2} \varrho^{n}$ for all $n \in \mathbb{N}$.

If the perturbation enjoys stronger regularity properties, then much sharper results can be obtained. Such results follow from a generalization of [13], along the lines of [17], that can be found in $\S 8$.

To keep the exposition simple let us restrict ourselves to deterministic perturbations. As $\mathcal{C}^{r+1}(X, X)$ has naturally the structure of a $\mathcal{C}^{r+1}$ Banach manifold, it makes sense to consider perturbations belonging to $\mathcal{C}^{s}\left([-1,1], \mathcal{C}^{r+1}(X, X)\right)$, that is curves $T_{t}$ of $\mathcal{C}^{r+1}$ maps from $X$ to $X$ such that, when viewed in coordinates, their first $s$ derivatives with respect to $t$ are $\mathcal{C}^{r+1}$ functions.

THEOREM 2.8. Let $q>0$ and $p, s \in \mathbb{N}^{*}$ be such that $p+q+s<r+1$. Let $T_{t} \in \mathcal{C}^{s}\left([-1,1], \mathcal{C}^{r+1}(X, X)\right)$ be such that $T_{0}$ is an Anosov diffeomorphism. Let $\varrho>\max \left(\lambda^{-p}, v^{q}\right)$. There exists $t_{*}>0$ such that, for all $t \in\left[-t_{*}, t_{*}\right]$, the eigenvalues and eigenprojectors $\lambda_{i}(t), \Pi_{i}(t)$ associated to $\mathcal{L}_{T_{t}}$ with $\left|\lambda_{i}(0)\right|>\varrho$ satisfy:

(1) if $\lambda_{i}(0)$ is simple, then $\lambda_{i}(t) \in \mathcal{C}^{s-1}$;

(2) $\Pi_{i}(t) \in \mathcal{C}^{s-1}\left(\mathcal{B}^{p-1+s, q}, \mathcal{B}^{p-1, q+s}\right)$.

The above theorem is proved in $\S 9$ by showing that the hypotheses of Theorem 8.1 hold in the present context. 
Remark 2.9. Note that, in Theorem 2.8, there is some limitation to the differentiability, coming from the fact that $p$ is an integer, namely $s<r$ (see also Remark 2.4). In certain cases this can yield a weaker result than [23] where, in the case $s=r+1$, it is proven that the eigenvalues are $\mathcal{C}^{r-1}$. Yet, [23] is limited to the peripheral eigenvalues and gives much less information on the eigenspaces.

Remark 2.10. Note that, although not explicitly stated, all the constants in Theorems 2.7 and 2.8 are constructive and can, at least in principle, be rigorously computed in specific examples (even though this would involve computer-assisted techniques; see [18] for a discussion of such issues).

Remark 2.11. Beside the eigenvalues and the eigenprojectors, the above theory implies results also for other physically relevant quantities. For example, if $T_{0}$ is a transitive Anosov map and $f \in \mathcal{C}^{r}$, let $f_{t}=f-\int f d \mu_{\mathrm{SRB}}\left(T_{t}\right) \dagger$. It is well known that $(1 / \sqrt{n}) \sum_{k=0}^{n-1} f_{t} \circ T_{t}^{k}$ converges in law to a Gaussian with zero mean and variance

$$
\sigma(t)^{2}=-\mu_{\mathrm{SRB}}\left(f_{t}^{2}\right)+2 \sum_{n=0}^{\infty} \mu_{\mathrm{SRB}}\left(f_{t} \circ T_{t}^{n} f_{t}\right)=-\mu_{\mathrm{SRB}}\left(f_{t}^{2}\right)+2\left\langle\left(\mathrm{Id}-\widetilde{\mathcal{L}}_{T_{t}}\right)^{-1} \mu_{t}, f_{t}\right\rangle
$$

where $\left\langle\mu_{t}, \varphi\right\rangle:=\left\langle\mu_{\mathrm{SRB}}, f_{t} \varphi\right\rangle$ and $\widetilde{\mathcal{L}}_{T_{t}}$ is the restriction of the operator $\mathcal{L}_{T_{t}}$ to the kernel of its eigenprojector for the eigenvalue 1, i.e. $\left\{h \in \mathcal{B}^{p, q}:\langle h, 1\rangle=0\right\}$. By the results of $\S \S 8$ and 9 it follows then that $\sigma \in \mathcal{C}^{s-1}$ and one can actually compute formulae for its Taylor expansion up to order $s-1$.

We conclude this section with a warning to the reader.

Remark 2.12. Through the paper we will use $C$ and $C_{\alpha}$ to designate generic constants depending only on the map, the Banach spaces and, eventually, on the parameter $\alpha$. Their actual numerical value can thus change from one occurrence to the next.

\section{Definition and properties of the admissible leaves}

Replacing the metric by an adapted metric following Mather [21], we can assume that the expansion of $D T(x)$ along the unstable directions is stronger than $\lambda$, the contraction along the stable directions is stronger than $v$, and the angle between the stable and unstable directions is everywhere arbitrarily close to $\pi / 2$. For small enough $\kappa$, we define the stable cone at $x \in X$ by

$$
\mathcal{C}(x)=\left\{u+v \in T_{x} X \mid u \in E^{s}(x), v \perp E^{s}(x),\|v\| \leq \kappa\|u\|\right\} .
$$

If $\kappa$ is small enough, $D T^{-1}(x)(\mathcal{C}(x) \backslash\{0\})$ is included in the interior of $\mathcal{C}\left(T^{-1} x\right)$, and $D T^{-1}(x)$ expands the vectors in $\mathcal{C}(x)$ by $v^{-1}$.

There exists a finite number of $\mathcal{C}^{\infty}$ coordinate charts $\psi_{1}, \ldots, \psi_{N}$ such that $\psi_{i}$ is defined on a subset $\left(-r_{i}, r_{i}\right)^{d}$ of $\mathbb{R}^{d}$ (with its standard Euclidian norm), such that:

(1) $D \psi_{i}(0)$ is an isometry;

(2) $D \psi_{i}(0) \cdot\left(\mathbb{R}^{d_{s}} \times\{0\}\right)=E^{s}\left(\psi_{i}(0)\right)$;

(3) the $\mathcal{C}^{r+1}$-norms of $\psi_{i}$ and its inverse are bounded by $1+\kappa$;

$\dagger$ By Theorem 2.3, $\mu_{\mathrm{SRB}}\left(T_{t}\right)$ is simply the eigenvector of $\mathcal{L}_{T_{t}}$ associated to the eigenvalue 1. 
(4) there exists $c_{i} \in(\kappa, 2 \kappa)$ such that the cone $\mathcal{C}_{i}=\left\{u+v \in \mathbb{R}^{d} \mid u \in \mathbb{R}^{d_{s}} \times\{0\}, v \in\right.$ $\left.\{0\} \times \mathbb{R}^{d_{u}},\|v\| \leq c_{i}\|u\|\right\}$ satisfies the following property: for any $x \in\left(-r_{i}, r_{i}\right)^{d}$, $D \psi_{i}(x) \mathcal{C}_{i} \supset \mathcal{C}\left(\psi_{i} x\right)$ and $D T^{-1}\left(D \psi_{i}(x) \mathcal{C}_{i}\right) \subset \mathcal{C}\left(T^{-1} \circ \psi_{i}(x)\right)$

(5) the manifold $X$ is covered by the open sets $\left(\psi_{i}\left(\left(-r_{i} / 2, r_{i} / 2\right)^{d}\right)\right)_{i=1, \ldots, N}$.

It is easy to construct such a chart around any point of $X$, hence a finite number of them is sufficient to cover the whole manifold by compactness.

Let $G_{i}(K)$ be the set of graphs of functions $\chi$ defined on a subset of $\left(-r_{i}, r_{i}\right)^{d_{s}}$ and taking values in $\left(-r_{i}, r_{i}\right)^{d_{u}}$, belonging to $\mathcal{C}^{r+1}$, with $|D \chi| \leq c_{i}$ (i.e. the tangent space to the graph of $\chi$ belongs to the cone $\mathcal{C}_{i}$ ) and with $|\chi|_{\mathcal{C}^{r+1}} \leq K \dagger$.

The following is a classical consequence of the uniform hyperbolicity of $T$.

LEMMA 3.1. If $K$ is large enough, then there exists $K^{\prime}<K$ such that, for any $W \in$ $G_{i}(K)$ and for any $1 \leq j \leq N$, the set $\psi_{j}^{-1} \circ T^{-1} \circ \psi_{i}(W)$ belongs to $G_{j}\left(K^{\prime}\right)$.

Until the end of the paper, we fix a constant $K$ satisfying the conclusion of Lemma 3.1.

If $\kappa$ is small enough, then $v^{-1}>(1+\kappa)^{2} \sqrt{1+4 \kappa^{2}}$. Hence, there exists $A>1$ such that

$$
\frac{v^{-1}}{(1+\kappa)^{2} \sqrt{1+4 \kappa^{2}}}(A-1)>A .
$$

Take $\delta>0$ small enough so that $A \delta<\min \left(r_{i}\right) / 6$.

We define an admissible graph as a map $\chi$ defined on some ball $\bar{B}(x, A \delta)$ included in $\left(-2 r_{i} / 3,2 r_{i} / 3\right)^{d_{s}}$, taking its values in $\left(-2 r_{i} / 3,2 r_{i} / 3\right)^{d_{u}}$, with range(Id, $\left.\chi\right) \in G_{i}(K)$. Denote by $\Xi_{i}$ the set of admissible graphs on $\left(-2 r_{i} / 3,2 r_{i} / 3\right)^{d_{s}}$.

Given an admissible graph $\chi \in \Xi_{i}$, we will call $\widetilde{W}:=\psi_{i} \circ(\operatorname{Id}, \chi)(\bar{B}(x, A \delta))$ the associated full admissible leaf and $W:=\psi_{i} \circ(\mathrm{Id}, \chi)(\bar{B}(x, \delta))$ the admissible leaf $\ddagger$.

Let

$$
\Sigma=\left\{\psi_{i} \circ(\operatorname{Id}, \chi)(\bar{B}(x, \delta)) \mid \chi: \bar{B}(x, A \delta) \rightarrow \mathbb{R}^{d_{u}} \text { belongs to } \Xi_{i}\right\} .
$$

This is the set of admissible leaves. Note that, by construction, they $\operatorname{are} \mathcal{C}^{r+1} \operatorname{disks} \mathcal{C}^{1}$ close to the stable manifolds.

We can use these admissible leaves to give another expression of the norm (2.2) in coordinates. For $h \in \mathcal{C}^{r}(X, \mathbb{R})$, set

$$
\|h\|_{p, q}^{\sim}=\sup _{\substack{|\alpha|=p \\ 1 \leq i \leq N}} \sup _{\substack{\chi: \bar{B}(x, A \delta) \rightarrow \mathbb{R}^{d_{u}} \\ \chi \in \Xi_{i}}} \sup _{\substack{\varphi \in \mathcal{C}_{0}^{q}(\bar{B}(x, \delta), \mathbb{R}) \\|\varphi|_{\mathcal{C}} \leq \leq 1}} \int_{B(x, \delta)}\left[\partial^{\alpha}\left(h \circ \psi_{i}\right)\right] \circ(\mathrm{Id}, \chi) \cdot \varphi
$$

and

$$
\|h\|_{p, q}^{\prime}=\sup _{0 \leq k \leq p}\|h\|_{k, q+k}^{\sim}=\sup _{p^{\prime} \leq p, q^{\prime} \geq q+p^{\prime}}\|h\|_{p^{\prime}, q^{\prime}}^{\sim}
$$

The following lemma proves that this norm is equivalent to the norm (2.2), and gives a little bit more that will be useful later.

$\dagger$ A function defined on an arbitrary subset $A$ of $\mathbb{R}^{d}$ is of class $\mathcal{C}^{r+1}$ if there exists a $\mathcal{C}^{r+1}$ extension to an open neighborhood of $A$. Its norm is the infimum of the norms of such extensions.

$¥$ Note that one can talk about an admissible leaf only if it is given by $\chi \in \Xi_{i}$, that is if there exists an associated full admissible leaf. 
LEMMA 3.2. If $p+q<r$, the norms $\|h\|_{p, q}$ and $\|h\|_{p, q}^{\prime}$ are equivalent on $\mathcal{C}^{r}(X, \mathbb{R})$. Moreover, there exists $C>0$ such that, for all $0 \leq k \leq p$, for all $f \in \mathcal{C}^{q+k}$,

$$
\sup _{W \in \Sigma} \sup _{\substack{v_{1}, \ldots, v_{k} \in \mathcal{V}^{q+k}(W) \\\left|v_{i}\right|_{\mathcal{C}^{q+k}} \leq 1}} \sup _{\substack{\varphi \in \mathcal{C}_{0}^{q+k}(W, \mathbb{R}) \\|\varphi|_{\mathcal{C}^{q+k}} \leq 1}} \int_{W} v_{1} \cdots v_{k}(f h) \cdot \varphi \leq C\|h\|_{p, q}^{\prime}|f|_{\mathcal{C}^{q+k}} .
$$

Proof. The inequality $\|h\|_{k, q+k}^{\sim} \leq C\|h\|_{k, q+k}^{-}$is trivial, as the images in the manifold of the coordinate vector fields have a bounded $\mathcal{C}^{r}$ norm. Hence, it is sufficient to prove (3.5) as, for $f=1$, it will imply $\|h\|_{k, q+k}^{-} \leq C\|h\|_{p, q}^{\prime}$.

We can without loss of generality work in one of the coordinate charts $\psi_{i}$. Let $\chi$ be an admissible graph, and let $v_{1}, \ldots, v_{k}$ be $\mathcal{C}^{q+k}$ vector fields on a neighborhood of the graph of $\chi$. Decomposing $v_{j}$ along the coordinate vector fields, we can assume that $v_{j}=f_{j} \partial_{\alpha(j)}$ where $f_{j} \in \mathcal{C}^{q+k},\left|f_{j}\right|_{\mathcal{C}^{q+k}} \leq 1$ and $\alpha(j) \in\{1, \ldots, d\}$.

In $v_{1} \cdots v_{k}(f h)$, the differential $\partial_{\alpha(j)}$ in $v_{j}$ can be used to differentiate either a function $f_{i}$ with $i>j$, or $f$, or $h$. For $1 \leq i \leq k$, denote by $J_{i}$ the set of the indices $j$ such that $\partial_{\alpha(j)}$ is used to differentiate $f_{i}$. It is a subset of $\{1, \ldots, i-1\}$ (in particular, $J_{1}=\emptyset$ ). Denote also by $J_{0}$ the set of indices used to differentiate $f$, and by $J$ the remaining indices, used to differentiate $h$. We obtain

$v_{1} \cdots v_{k}(f h)=\sum_{J, J_{0}, J_{1}, \ldots, J_{k}}\left(\prod_{j \in J} \partial_{\alpha(j)} h\right)\left(\prod_{j \in J_{0}} \partial_{\alpha(j)} f\right)\left(\prod_{j \in J_{1}} \partial_{\alpha(j)} f_{1}\right) \cdots\left(\prod_{j \in J_{k}} \partial_{\alpha(j)} f_{k}\right)$,

where we sum over all partitions $J, J_{0}, J_{1}, \ldots, J_{k}$ of the set $\{1, \ldots, k\}$ such that $J_{i} \subset$ $\{1, \ldots, i-1\}$ for $1 \leq i \leq k$. Each term

$$
\int\left[\left(\prod_{j \in J} \partial_{\alpha(j)} h\right)\left(\prod_{j \in J_{0}} \partial_{\alpha(j)} f\right)\left(\prod_{j \in J_{1}} \partial_{\alpha(j)} f_{1}\right) \cdots\left(\prod_{j \in J_{k}} \partial_{\alpha(j)} f_{k}\right)\right] \circ(\mathrm{Id}, \chi) \cdot \varphi
$$

is an integral of $p^{\prime}=|J|$ derivatives of $h$ against a test function of differentiability $\mathcal{C}^{q^{\prime}}$ where $q^{\prime}=\min _{0 \leq s \leq k}\left(q+k-\left|J_{s}\right|\right) \geq q+|J|$. As $p^{\prime} \leq p$ and $q^{\prime} \geq q+p^{\prime}$, it is bounded by $C\|h\|_{p, q}^{\prime}|f|_{\mathcal{C}^{q+k}}$ by (3.4).

In the following, we will work interchangeably with one expression of the norm or the other and we will suppress the 'prime' unless this creates confusion.

The reason for integrating in (3.3) only on admissible leaves, rather than on full admissible leaves, is that the preimage of an admissible leaf can be covered by a finite number of admissible leaves. We will in fact need a slightly more precise result, conveniently expressed in terms of the following notion. For $\gamma>1$, a $\gamma$-admissible graph is a map defined on a ball $\bar{B}(x, \gamma A \delta) \subset\left(-2 r_{i} / 3 \gamma, 2 r_{i} / 3 \gamma\right)^{d_{s}}$, taking its values in $\left(-2 r_{i} / 3 \gamma, 2 r_{i} / 3 \gamma\right)^{d_{u}}$, whose graph belongs to $G_{i}(K)$. The corresponding $\gamma$-admissible leaf is $\psi_{i} \circ(\mathrm{Id}, \chi)(\bar{B}(x, \delta / \gamma))$.

LEMMA 3.3. There exists $\gamma_{0}>1$ satisfying the following property: for any full admissible leaf $\widetilde{W}$ and $n \in \mathbb{N}^{*}$, for any $1 \leq \gamma \leq \gamma_{0}$, there exist $\gamma$-admissible leaves $W_{1}, \ldots, W_{\ell}$, whose number $\ell$ is bounded by a constant depending only on $n$, such that:

(1) $T^{-n}(W) \subset \bigcup_{j=1}^{\ell} W_{j}$;

(2) $T^{-n}(\tilde{W}) \supset \bigcup_{j=1}^{\ell} W_{j}$; 
(3) there exists a constant $C$ (independent of $W$ and $n$ ) such that a point of $T^{-n} \widetilde{W}$ is contained in at most $C$ sets $W_{j}$;

(4) there exist functions $\rho_{1}, \ldots, \rho_{\ell}$ of class $\mathcal{C}^{r+1}$ and compactly supported on $W_{j}$ such that $\sum \rho_{j}=1$ on $T^{-n}(W)$, and $\left|\rho_{j}\right|_{\mathcal{C}^{r+1}} \leq C$.

Proof. Let $\chi: \bar{B}(x, A \delta) \rightarrow\left(-2 r_{i} / 3,2 r_{i} / 3\right)^{d_{u}}$ be an admissible graph. Let $W=$ $\psi_{i} \circ(\operatorname{Id}, \chi)(\bar{B}(x, \delta))$ be the admissible leaf corresponding to $\chi$, and $\widetilde{W}$ the corresponding full admissible leaf.

Take $y \in \bar{B}(x, \delta)$ (so that $\bar{B}(y,(A-1) \delta) \subset \bar{B}(x, A \delta)$ ) and $j$ such that $T^{-n}$ 。 $\psi_{i}(y, \chi(y)) \in \psi_{j}\left(\left(-r_{j} / 2, r_{j} / 2\right)^{d}\right)$. Let $\pi: \mathbb{R}^{d} \rightarrow \mathbb{R}^{d_{s}}$ be the projection onto the first components. The map $T^{-n}$ expands the distances by at least $v^{-n}$ along $\widetilde{W}$. The maps $\psi_{i}^{-1}$ and $\psi_{j}$ are $(1+\kappa)$-Lipschitz and $|\pi(v)| \geq\left(1 / \sqrt{1+4 \kappa^{2}}\right)|v|$ when the vector $v$ points in a stable cone $\mathcal{C}_{j}$. Hence, the map $F:=\pi \circ \psi_{j}^{-1} \circ T^{-n} \circ \psi_{i} \circ(\mathrm{Id}, \chi)$ expands the distances by at least $v^{-n} /\left[(1+\kappa)^{2} \sqrt{1+4 \kappa^{2}}\right]$. If $\gamma$ is close enough to 1 , then (3.1) implies that the image by $F$ of the ball $\bar{B}(y,(A-1) \delta)$ contains the ball $\bar{B}(F(y), \gamma A \delta)$. We can then define a map $\chi_{F(y)}: \bar{B}(F(y), \gamma A \delta) \rightarrow\left(-2 r_{j} /(3 \gamma), 2 r_{j} /(3 \gamma)\right)^{d_{u}}$ such that its graph is contained in $\psi_{j}^{-1}\left(T^{-n} \tilde{W}\right)$. In particular, $\chi_{F(y)}$ is a $\gamma$-admissible graph, by Lemma 3.1.

We have shown that $T^{-n} W$ can be covered by $\gamma$-admissible leaves. As the $\mathcal{C}^{r+1}$ norm of admissible leaves is uniformly bounded, we can then apply general results on the existence of good partitions of unity, such as [11, Theorem 1.4.10], to finish the proof of the lemma.

Remark 3.4. We will mostly use this lemma with $\gamma=1$, to get a covering of $T^{-n} W$ by admissible leaves. However, in the study of perturbations of $T$, we will need to use some $\gamma>1$.

\section{Description of the space $\mathcal{B}^{p, q}$}

Take a covering of $X$ by sets of diameter at most $\delta$ and a partition of unity subordinated to this covering. Using admissible leaves supported in each of these sets, we easily check that there exists a constant $C$ such that, for all $h \in \mathcal{C}^{r}(X, \mathbb{R})$ and for all $\varphi \in \mathcal{C}^{q}(X, \mathbb{R})$,

$$
\left|\int_{X} h \cdot \varphi\right| \leq C\|h\|_{p, q}|\varphi|_{\mathcal{C} q} .
$$

Passing to the completion, we obtain that any $h \in \mathcal{B}^{p, q}$ gives a distribution on $X$ of order at most $q \dagger$. Denote by $\mathcal{D}_{q}^{\prime}$ the set of distributions of order at most $q$ with its canonical norm.

Proposition 4.1. The map $\mathcal{B}^{p, q} \rightarrow \mathcal{D}_{q}^{\prime}$ is a continuous injection.

Proof. The continuity is trivial from the previous remarks.

Take $h \in \mathcal{C}^{r}(X, \mathbb{R})$. Let $\chi: \bar{B}(x, A \delta) \rightarrow\left(-2 r_{i} / 3,2 r_{i} / 3\right)^{d_{u}}$ be an admissible graph and let $\alpha$ be a multi-index with $|\alpha| \leq p$. We can define a distribution $D_{\alpha, \chi}(h)$ of order $q+|\alpha|$, on the ball $B(0, \delta)$, setting $\left\langle D_{\alpha, \chi}(h), \varphi\right\rangle=\int_{B(0, \delta)} \partial^{\alpha}\left(h \circ \psi_{i}\right)(x+\eta, \chi(x+\eta)) \cdot \varphi(\eta) d \eta$. The map $h \mapsto D_{\alpha, \chi}(h)$ is continuous for the $\|\cdot\|_{p, q}$-norm, and hence it can be extended to

$\dagger$ Here, we are using the fact that there is a canonical measure on $X$, namely the Riemannian measure. Otherwise, we would have to distinguish between generalized functions and generalized densities. 
the space $\mathcal{B}^{p, q}$. The norm of an element $h$ of $\mathcal{B}^{p, q}$ is by definition equal to the supremum of the norms of the corresponding distributions $D_{\alpha, \chi}(h)$.

Assume that $\partial^{\alpha}=\partial_{j} \partial^{\beta}$. Let $\chi_{\varepsilon}$ be the admissible graph obtained by translating the graph of $\chi$ by $\varepsilon$ in the direction $x_{j}$. For $h \in \mathcal{C}^{r}$, the map $\varepsilon \mapsto D_{\alpha, \chi_{\varepsilon}}(h)$ is continuous. By density, it is continuous for any $h \in \mathcal{B}^{p, q}$. Moreover,

$$
D_{\beta, \chi_{\varepsilon}}(h)-D_{\beta, \chi}(h)=\varepsilon \int_{0}^{1} D_{\alpha, \chi_{t \varepsilon}}(h) d t .
$$

As $\varepsilon \mapsto D_{\alpha, \chi_{\varepsilon}}(h)$ is continuous, we obtain that, for any $h \in \mathcal{B}^{p, q}$,

$$
D_{\alpha, \chi}(h)=\lim _{\varepsilon \rightarrow 0} \frac{D_{\beta, \chi_{\varepsilon}}(h)-D_{\beta, \chi}(h)}{\varepsilon} .
$$

Take $h \in \mathcal{B}^{p, q}$ different from zero. Then there exists an admissible graph $\chi$ such that $D_{\emptyset, \chi}(h) \neq 0$ : otherwise, (4.1) would imply that all the distributions $D_{\alpha, \chi}(h)$ vanish, which means that $h=0$ in $\mathcal{B}^{p, q}$. As $\mathcal{C}^{\infty}$ is dense in $\mathcal{C}^{q}$, there exists $\varphi \in \mathcal{C}^{\infty}$ such that $\left\langle D_{\emptyset, \chi}(h), \varphi\right\rangle \neq 0$. Then, for any $\chi^{\prime}$ close enough to $\chi$, we still have $\left\langle D_{\emptyset, \chi^{\prime}}(h), \varphi\right\rangle \neq 0$ by continuity. Hence, we can construct a $\mathcal{C}^{\infty}$ function $\tilde{\varphi}$ supported on a neighborhood of the graph of $\chi$ such that $\langle h, \widetilde{\varphi}\rangle \neq 0$. Therefore, the distribution given by $h$ is non-zero.

Remark 4.2. There exist canonically defined maps $\mathcal{B}^{p, q} \rightarrow \mathcal{B}^{p-1, q}$ and $\mathcal{B}^{p, q} \rightarrow \mathcal{B}^{p, q^{\prime}}$ for $q^{\prime}>q$, obtained by extending continuously the canonical embedding of $\mathcal{C}^{r}$ functions. Proposition 4.1 implies, in particular, that these maps are injective.

Remark 4.3. When $h$ is $\mathcal{C}^{r}$ and $p+q<r$, then $\|h\|_{p, q} \leq C|h|_{\mathcal{C}^{r}}$. Hence, the embedding of $\mathcal{C}^{r}(X, \mathbb{R})$ in $\mathcal{B}^{p, q}$ is continuous. As $\mathcal{C}^{\infty}(X, \mathbb{R})$ is dense in $\mathcal{C}^{r}(X, \mathbb{R})$ for the $\mathcal{C}^{r}$-norm, it implies that $\mathcal{C}^{\infty}(X, \mathbb{R})$ is dense in $\mathcal{B}^{p, q}$. Hence, we could also have obtained $\mathcal{B}^{p, q}$ by completing $\mathcal{C}^{\infty}(X, \mathbb{R})$.

It is interesting to give explicit examples of non-trivial elements of $\mathcal{B}^{p, q}$.

Proposition 4.4. Let $W$ be a $\mathcal{C}^{p+1}$-submanifold of dimension $d_{u}$ everywhere transverse to the cones $D \psi_{i}\left(\mathcal{C}_{i}\right)$ (e.g. a piece of unstable manifold) and $\mu$ a $\mathcal{C}^{p}$-density on $W$, with compact support. Then the distribution $\ell(\varphi):=\int_{W} \varphi d \mu$ belongs to $\mathcal{B}^{p, q}$.

Proof. Without loss of generality we can assume that the manifold $W$ belongs to one chart $\left(\left(-r_{i}, r_{i}\right)^{d}, \psi_{i}\right)$. We will work only in such a chart, and omit the coordinate change $\psi_{i}$. The pullback in this chart of the Riemannian measure is of the form $\gamma(\eta, \xi) d \eta d \xi$, where $(\eta, \xi) \in \mathbb{R}^{d_{s}} \times \mathbb{R}^{d_{u}}$.

The manifold $W$ is given by the graph of a $\mathcal{C}^{p+1}$ function $\zeta: \mathbb{R}^{d_{u}} \rightarrow \mathbb{R}^{d_{s}}$. The density of $\mu$ is then given by a $\mathcal{C}^{p}$ function $f: \mathbb{R}^{d_{u}} \rightarrow \mathbb{R}$ with compact support.

Let $f_{\varepsilon} \in \mathcal{C}^{\infty}$ be such that $\left|f-f_{\varepsilon}\right|_{\mathcal{C}} \leq \leq \varepsilon$. Take also $\zeta_{\varepsilon} \in \mathcal{C}^{\infty}$ with $\left|\zeta-\zeta_{\varepsilon}\right|_{\mathcal{C}^{p+1}} \leq \varepsilon$. Let $\vartheta: \mathbb{R}^{d_{s}} \rightarrow \mathbb{R}_{+}$be a $\mathcal{C}^{\infty}$ function supported in $B(0,1)$ and with $\int \vartheta=1$. Set $\vartheta_{\varepsilon}(\eta)=\left(1 / \varepsilon^{d_{s}}\right) \vartheta(\eta / \varepsilon)$ : it is supported in $B(0, \varepsilon)$ and has integral one. Finally, let $h_{\varepsilon}(\eta, \xi)=\vartheta_{\varepsilon}\left(\eta-\zeta_{\varepsilon}(\xi)\right) f_{\varepsilon}(\xi) \gamma(\eta, \xi)^{-1}$ : it is a $\mathcal{C}^{\infty}$ function, and the corresponding distribution in $\mathcal{D}_{q}^{\prime}$ is given by

$$
\varphi \mapsto \int h_{\varepsilon}(\eta, \xi) \varphi(\eta, \xi) \gamma(\eta, \xi) d \eta d \xi=\int \vartheta_{\varepsilon}\left(\eta-\zeta_{\varepsilon}(\xi)\right) f_{\varepsilon}(\xi) \varphi(\eta, \xi) d \eta d \xi
$$


When $\varepsilon \rightarrow 0$, this distribution converges to $\ell$. Hence, the result will be proved if we show that $\left\{h_{\varepsilon}\right\}$ is a Cauchy sequence in $\mathcal{B}^{p, q}$.

Take $\alpha$ with $|\alpha| \leq p$. Then one has

$$
\partial^{\alpha} h_{\varepsilon}(\eta, \xi)=\sum_{\beta \leq \alpha}\left(\partial^{\beta} \vartheta_{\varepsilon}\right)\left(\eta-\zeta_{\varepsilon}(\xi)\right) g_{\alpha, \beta, \varepsilon}(\xi),
$$

where the function $g_{\alpha, \beta, \varepsilon}$ is in $\mathcal{C}^{\infty}$ and converges in $\mathcal{C}^{p-|\alpha|+|\beta|}$ to a function $g_{\alpha, \beta, 0}$ when $\varepsilon \rightarrow 0$.

Let $\chi$ be an admissible graph and $\varphi$ a $\mathcal{C}^{q+|\alpha|}$ test function with $|\varphi|_{\mathcal{C}^{q+|\alpha|}} \leq 1$. Then

$$
\int \varphi(\eta) \partial^{\alpha} h_{\varepsilon}(\eta, \chi(\eta))=\sum_{\beta} \int \varphi(\eta)\left(\partial^{\beta} \vartheta_{\varepsilon}\right)\left(\eta-\zeta_{\varepsilon}(\chi(\eta)) g_{\alpha, \beta, \varepsilon}(\chi(\eta))\right) .
$$

As $W$ is everywhere transverse to the cone $\mathcal{C}_{i}$, the map $\theta_{\varepsilon}: \eta \mapsto \eta-\zeta_{\varepsilon}(\chi(\eta))$ is a $\mathcal{C}^{p+1}$ diffeomorphism, and it converges when $\varepsilon \rightarrow 0$. Using this change of coordinates in (4.2), integrating by parts and given the fact that $\vartheta_{\varepsilon}$ is a $\mathcal{C}^{\infty}$ mollifier, we obtain that (4.2) converges when $\varepsilon \rightarrow 0$. Moreover, the speed of convergence is independent of the graph $\chi$ or the test function $\varphi$, as all norms are uniformly bounded. Hence, $\left\|h_{\varepsilon}-h_{\mathcal{\varepsilon}^{\prime}}\right\|_{p, q} \rightarrow 0$ when $\varepsilon, \varepsilon^{\prime} \rightarrow 0$, i.e. $h_{\varepsilon}$ is a Cauchy sequence in $\mathcal{B}^{p, q}$.

\section{Compactness}

This section is devoted to the proof of Lemma 2.1. We will work only in coordinate charts, using in an essential way the linear structure to interpolate between admissible leaves.

Let $h \in \mathcal{C}^{r}$. Fix $\varepsilon>0$. Take $1 \leq i \leq N$. As $p+q<r$, the injection $\mathcal{C}^{r+1} \rightarrow \mathcal{C}^{p+q}$ is compact. Therefore, there exists a finite number of admissible graphs $\chi_{1}, \ldots, \chi_{s}$ defined on balls $\bar{B}\left(x_{1}, A \delta\right), \ldots, \bar{B}\left(x_{s}, A \delta\right)$ such that any other admissible graph $\chi$ defined on a ball $\bar{B}(x, A \delta)$ is at a distance at most $\varepsilon$ of some $\chi_{j}$, in the sense that $\left|x-x_{j}\right| \leq \varepsilon$ and $\left|\eta \mapsto \chi(x+\eta)-\chi_{j}\left(x_{j}+\eta\right)\right|_{\mathcal{C} p+q\left(\bar{B}(0, \delta), \mathbb{R}^{d_{u}}\right)} \leq \varepsilon$.

Take $\alpha$ with $|\alpha|=p-1$ and $\varphi \in \mathcal{C}_{0}^{p+q}(\bar{B}(0, \delta), \mathbb{R})$ with $|\varphi|_{\mathcal{C}^{p+q}} \leq 1$. Write

$$
f_{t}(\eta)=\left(x_{j}+\eta+t\left(x-x_{j}\right), \chi_{j}\left(x_{j}+\eta\right)+t\left(\chi(x+\eta)-\chi_{j}\left(x_{j}+\eta\right)\right)\right) .
$$

Write also $F(z)=\partial^{\alpha}\left(h \circ \psi_{i}\right)(z)$. Then, for $\eta \in B(0, \delta)$,

$$
\begin{aligned}
\partial^{\alpha}\left(h \circ \psi_{i}\right)(x+\eta, \chi(x+\eta))-\partial^{\alpha}\left(h \circ \psi_{i}\right)\left(x_{j}+\eta, \chi_{j}\left(x_{j}+\eta\right)\right) \\
\quad=F\left(f_{1}(\eta)\right)-F\left(f_{0}(\eta)\right) \\
\quad=\int_{0}^{1} D F\left(f_{t}(\eta)\right) \cdot\left(x-x_{j}, \chi(x+\eta)-\chi_{j}\left(x_{j}+\eta\right)\right) d t .
\end{aligned}
$$

Hence,

$$
\begin{gathered}
\int \partial^{\alpha}\left(h \circ \psi_{i}\right)(x+\eta, \chi(x+\eta)) \varphi(\eta) d \eta-\int \partial^{\alpha}\left(h \circ \psi_{i}\right)\left(x_{j}+\eta, \chi_{j}\left(x_{j}+\eta\right)\right) \varphi(\eta) d \eta \\
=\int_{0}^{1}\left(\int D F\left(f_{t}(\eta)\right) \cdot\left(x-x_{j}, \chi(x+\eta)-\chi_{j}\left(x_{j}+\eta\right)\right) \varphi(\eta) d \eta\right) d t .
\end{gathered}
$$

When $t$ is fixed, the last integral is an integral along the graph given by $f_{t}$. This graph is admissible as it is an interpolation between two admissible graphs (here, the fact that the 
cone $\mathcal{C}_{i}$ is constant is essential). As $\left|x-x_{j}\right| \leq \varepsilon$ and $\left|\chi(x+\eta)-\chi_{j}\left(x_{j}+\eta\right)\right|_{\mathcal{C}^{p+q}} \leq \varepsilon$, this term can be estimated by $C \varepsilon\|h\|_{p, q+p}$. We have proved that

$$
\begin{aligned}
& \|h\|_{p-1, q+p}^{\sim} \\
& \quad=\sup _{|\alpha|=p} \sup _{\substack{\chi: \bar{B}(x, A \delta) \rightarrow \mathbb{R}^{d_{u}} \\
\chi \in \Xi_{i}}} \sup _{\varphi \in \mathcal{C}_{0}^{p+q}(\bar{B}(x, \delta), \mathbb{R})} \int_{B(x, \delta)} \partial^{\alpha}\left(h \circ \psi_{i}\right) \circ(\mathrm{Id}, \chi) \cdot \varphi \\
& \quad \leq C \varepsilon\|h\|_{p, q+p}^{\sim}+\sup _{|\alpha|=p} \sup _{\substack{\left.\left|\leq k \leq s \\
1 \leq k \in \mathcal{C}^{p+q}\left(\bar{B}\left(x_{k}, \delta\right), \mathbb{R}\right)\\
\right| \varphi\right|^{p+q} \leq 1}} \int_{B\left(x_{k}, \delta\right)} \partial^{\alpha}\left(h \circ \psi_{i}\right) \circ\left(\operatorname{Id}, \chi_{k}\right) \cdot \varphi,
\end{aligned}
$$

i.e. we have only a finite number of admissible graphs to consider.

In the following, we work with one graph $\chi=\chi_{k}$. The set of functions $\varphi \in$ $\mathcal{C}_{0}^{p+q}\left(\bar{B}\left(x_{k}, \delta\right), \mathbb{R}\right)$ with $|\varphi|_{\mathcal{C}^{p+q} \leq 1}$ is relatively compact for the $\mathcal{C}^{p+q-1}$ topology. Hence, there exists a finite set of functions $\varphi_{1}, \ldots, \varphi_{k}$ which are $\varepsilon$-dense. For any $\varphi$ as above, there exists $j$ such that $\left|\varphi-\varphi_{j}\right|_{\mathcal{C}^{p+q-1}} \leq \varepsilon$. As $\|h\|_{p-1, q+p-1}^{\sim} \leq\|h\|_{p, q}$,

$$
\int \partial^{\alpha}\left(h \circ \psi_{i}\right) \circ(\mathrm{Id}, \chi) \cdot \varphi \leq \int \partial^{\alpha}\left(h \circ \psi_{i}\right) \circ(\mathrm{Id}, \chi) \cdot \varphi_{j}+C \varepsilon\|h\|_{p, q} .
$$

To summarize, we have proved the existence of a finite number of continuous linear forms $v_{1}, \ldots, v_{\ell}$ on $\mathcal{B}^{p, q}$ such that, for any $h \in \mathcal{C}^{r}$,

$$
\|h\|_{p-1, q+p}^{\sim} \leq C \varepsilon\|h\|_{p, q}+\sup \left|v_{i}(h)\right| .
$$

By density of $\mathcal{C}^{r}$, this inequality is true for any $h \in \mathcal{B}^{p, q}$. This immediately implies the compactness we are looking for.

\section{Lasota-Yorke-type inequality}

This section is devoted to the proof of Lemma 2.2.

6.1. Proof of inequality (2.3). By density it suffices to prove it for $h \in \mathcal{C}^{r}$.

Take $W \in \Sigma$ and $n \in \mathbb{N}^{*}$. Let $\varphi \in \mathcal{C}_{0}^{q}(W, \mathbb{R})$ satisfy $|\varphi|_{\mathcal{C}^{q}} \leq 1$. Let $\rho_{1}, \ldots, \rho_{\ell}$ be the partition of unity on $T^{-n} W$ given by Lemma 3.3 (for $\gamma=1$ ), and $W_{1}, \ldots, W_{\ell}$ the corresponding admissible leaves. Let $h_{n}=h \cdot\left|\operatorname{det} D T^{n}\right|^{-1}$, then

$$
\int_{W} \mathcal{L}^{n} h \cdot \varphi=\int_{T^{-n} W} h_{n} \cdot \varphi \circ T^{n} \cdot J_{W} T^{n}
$$

where $J_{W} T^{n}$ is the Jacobian of $T^{n}: T^{-n} W \rightarrow W$. Using the partition of unity,

$$
\int_{W} \mathcal{L}^{n} h \cdot \varphi=\sum_{j=1}^{\ell} \int_{W_{j}} h_{n} \cdot \varphi \circ T^{n} \cdot J_{W} T^{n} \cdot \rho_{j} .
$$

The function $\varphi_{j}:=\varphi \circ T^{n} \cdot \rho_{j}$ is compactly supported on the admissible leaf $W_{j}$, and belongs to $\mathcal{C}^{q}$. Using the definition of the $\|\cdot\|_{0, q}$ norm along $W_{j}$ yields

$$
\left|\int_{W_{j}} h_{n} \cdot \varphi_{j} \cdot J_{W} T^{n}\right| \leq\left. C\|h\|_{0, q}|| \operatorname{det} D T^{n}\right|^{-1} \cdot \varphi_{j} \cdot J_{W} T^{n} \mid \mathcal{C}^{q}\left(W_{j}\right) .
$$

We will use repeatedly the following distortion lemmas. 
LEMMA 6.1. Let $\widetilde{W}$ be a full admissible leaf and $W^{\prime}$ an admissible leaf contained in $T^{-n} \widetilde{W}$. Let $1 \leq s \leq r$. Let $g_{0}, \ldots, g_{n-1}$ be strictly positive $\mathcal{C}^{s}$ functions on $W^{\prime}, \ldots, T^{n-1}\left(W^{\prime}\right)$ and let $L>0$ be such that, for any $x \in T^{i}\left(W^{\prime}\right)$, the $\mathcal{C}^{s}$ norm of $g_{i}$ is bounded on a neighborhood of $x$ by $L_{i}(x)$. Then

$$
\text { for all } x \in W^{\prime}, \quad\left|\prod_{i=0}^{n-1} g_{i} \circ T^{i}\right|_{\mathcal{C}^{s}\left(W^{\prime}\right)} \leq C e^{C L} \prod_{i=0}^{n-1} g_{i} \circ T^{i}(x)
$$

for some constant $C$ depending only on the map $T$.

Proof. Using the assumption on the $\mathcal{C}^{s}$ norm of $g_{i}$ and the uniform contraction of $T$ along $W^{\prime}$, it is easy to check that $\left|\prod_{i=0}^{n-1} g_{i} \circ T^{i}\right|_{\mathcal{C}^{s}\left(W^{\prime}\right)} \leq C L^{s}\left|\prod_{i=0}^{n-1} g_{i} \circ T^{i}\right|_{\mathcal{C}^{0}\left(W^{\prime}\right)}$.

The differential of the function $\log \left(\prod_{i=0}^{n-1} g_{i} \circ T^{i}\right)$ is also bounded by $C L$, from which, for any $x, y \in W^{\prime}$,

$$
\prod_{i=0}^{n-1} g_{i} \circ T^{i}(x) \leq C e^{C L} \prod_{i=0}^{n-1} g_{i} \circ T^{i}(y)
$$

LEMma 6.2. There exists $C>0$ such that, for each $n \in \mathbb{N}$, we have that

$$
\left.\sum_{j \leq \ell}|| \operatorname{det} D T^{n}\right|^{-1}\left|\mathcal{C}^{r}\left(W_{j}\right) \cdot\right| J_{W} T^{n} \mid \mathcal{C}^{r}\left(W_{j}\right) \leq C .
$$

Proof. Lemma 6.1 applies to estimate $\left.|| \operatorname{det} D T^{n}\right|^{-1} \mid \mathcal{C}_{\left(W_{j}\right)}$ and $\left|J_{W} T^{n}\right|_{\mathcal{C}^{r}\left(W_{j}\right)}$. For any $x \in W_{j}$,

$$
\left.\left.|| \operatorname{det} D T^{n}\right|^{-1}\left|\mathcal{C}^{r}\left(W_{j}\right) \cdot\right| J_{W} T^{n}\left|\mathcal{C}^{r}\left(W_{j}\right) \leq C\right| \operatorname{det} D T^{n}\right|^{-1}(x) J_{W} T^{n}(x) .
$$

In particular,

$$
\begin{aligned}
& \|\left.\operatorname{det} D T^{n}\right|^{-1}\left|\mathcal{C}^{r}\left(W_{j}\right) \cdot\right| J_{W} T^{n} \mid \mathcal{C}^{r}\left(W_{j}\right) \\
& \quad \leq C \int_{W_{j}}\left|\operatorname{det} D T^{n}\right|^{-1} J_{W} T^{n}=C \int_{T^{n}\left(W_{j}\right)}\left|\operatorname{det}\left(D T^{-n}\right)\right| .
\end{aligned}
$$

By Lemma 3.3, the sets $T^{n}\left(W_{j}\right)$ are contained in $\widetilde{W}$ and have a bounded number of overlaps. Let us consider the thickening $Z:=\bigcup_{x \in \widetilde{W}} W_{\rho}^{u}(x)$, where $W_{\rho}^{u}(x)$ is the ball of size $\rho$ in the unstable manifold through $x$. By usual distortion estimates,

$$
\begin{aligned}
\sum_{j \leq \ell} \int_{T^{n}\left(W_{j}\right)}\left|\operatorname{det}\left(D T^{-n}\right)\right| & \leq C \int_{\widetilde{W}}\left|\operatorname{det}\left(D T^{-n}\right)\right| \\
& \leq C \rho^{-d_{u}} \int_{Z}\left|\operatorname{det} D T^{-n}\right|=C \operatorname{Vol}\left(T^{-n} Z\right) \leq C .
\end{aligned}
$$

As $T^{n}$ is uniformly contracting along $T^{-n}(W)$, we have $\left|\varphi \circ T^{n}\right|_{\mathcal{C}^{q}\left(W_{j}\right)} \leq C|\varphi|_{\mathcal{C}^{q}} \leq C$, and $\left|\rho_{j}\right|_{\mathcal{C}^{q}\left(W_{j}\right)}$ is uniformly bounded by Lemma 3.3. This, together with (6.1) and (6.2) and Lemma 6.2, concludes the proof of inequality (2.3). 
6.2. Proof of inequality (2.4). Let $p \in \mathbb{N}$ and $q \geq 0$ satisfy $p+q<r$.

LEMMA 6.3. There exists a constant $C$ such that, for each $n \in \mathbb{N}$, there exists $C_{n}>0$ such that, for any $h \in \mathcal{B}^{p, q}$

$$
\text { for all } 0 \leq t<p,\left\|\mathcal{L}^{n} h\right\|_{t, q+t}^{-} \leq C\left(v^{q}\right)^{n}\|h\|_{p, q}+C_{n}\|h\|_{p-1, q+1}
$$

and

$$
\left\|\mathcal{L}^{n} h\right\|_{p, q+p}^{-} \leq C \max \left(\lambda^{-p}, v^{q}\right)^{n}\|h\|_{p, q}+C_{n}\|h\|_{p-1, q+1}
$$

Proof. We prove the lemma by induction over $t$. So, take $0 \leq t \leq p$ and assume that the conclusion of the lemma holds for all $t^{\prime}<t$. By density, it suffices to prove the lemma for $h \in \mathcal{C}^{r}$.

Let $W$ and $\widetilde{W}$ be an admissible leaf and the corresponding full admissible leaf. As before, we will use Lemma 3.3 (with $\gamma=1$ ) to write $T^{-n} W \subset \bigcup_{j} W_{j}$ and denote by $\rho_{j}$ the corresponding partition of unity given by Lemma 3.3.

Let $v_{1}, \ldots, v_{t} \in \mathcal{V}^{r}(W)$ with $\left|v_{i}\right|_{\mathcal{C}^{r}} \leq 1$, and let $\varphi \in \mathcal{C}_{0}^{t+q}(W)$ with $|\varphi|_{\mathcal{C}^{t+q}(W)} \leq 1$. Writing $h_{n}=h \cdot\left|\operatorname{det} D T^{n}\right|^{-1}$ as above, we want to prove that

$$
\left|\int_{W} v_{1} \cdots v_{t}\left(h_{n} \circ T^{-n}\right) \varphi\right| \leq \begin{cases}C\left(v^{q}\right)^{n}\|h\|_{p, q}+C_{n}\|h\|_{p-1, q+1} & \text { if } t<p, \\ C \max \left(\lambda^{-p}, v^{q}\right)^{n}\|h\|_{p, q}+C_{n}\|h\|_{p-1, q+1} & \text { if } t=p .\end{cases}
$$

The main idea of the proof will be to decompose each $v_{i}$ as a sum $v_{i}=w_{i}^{u}+w_{i}^{s}$ where $w_{i}^{s}$ is tangent to $W$, and $w_{i}^{u}$ 'almost' in the unstable direction. We will then get rid of $w_{i}^{s}$ by an integration by parts, and finish the proof by using the fact that $w_{i}^{u}$ is contracted by $D T^{-n}$.

Let $\Psi$ and $\Psi_{j}$ be coordinates charts with uniformly bounded $\mathcal{C}^{r+1}$ norms such that the images in the charts of $\widetilde{W}$ and $\widetilde{W}_{j}$ are contained in $\mathbb{R}^{d_{s}} \times\{0\}$. Let $\bar{v}_{i}(y)=$ $D \Psi\left(\Psi^{-1} y\right) v_{i}\left(\Psi^{-1} y\right)$, it has still a bounded $\mathcal{C}^{r}$ norm. The integral in (6.3) can be written as

$$
\int_{\Psi(W)} \bar{v}_{1} \cdots \bar{v}_{t}\left(h_{n} \circ T^{-n} \circ \Psi^{-1}\right) \cdot \varphi \circ \Psi^{-1} \cdot \operatorname{Jac}\left(\Psi^{-1}\right)
$$

We will work in this coordinate chart and, with a small abuse of notations, omit $\Psi$ in the formulas. As $\operatorname{Jac}\left(\Psi^{-1}\right)$ has a bounded $\mathcal{C}^{r}$ norm, we may also replace $\varphi$ with $\varphi \circ \Psi^{-1} \cdot \operatorname{Jac}\left(\Psi^{-1}\right)$. We will also work in the charts $\Psi_{j}$, and omit them as well in the formulas.

Remark 6.4. Note that, in the coordinate charts $\Psi, \Psi_{j}$ the manifolds $W, W_{j}$ are $\mathcal{C}^{\infty}$. This will be used extensively in the following.

Without loss of generality, we can assume that each $\bar{v}_{i}$ is of the form $f_{i} \partial_{\alpha(i)}$ where $f_{i}$ is bounded in $\mathcal{C}^{r}$ and $\partial_{\alpha(i)}$ is one of the coordinate vector fields. In $\bar{v}_{1} \ldots \bar{v}_{t}\left(h_{n} \circ T^{-n}\right)$, if we differentiate at least one of the functions $f_{i}$, we obtain an integral of $t^{\prime}<t$ derivatives of $h_{n} \circ T^{-n}$ against a function in $\mathcal{C}^{q+t^{\prime}}$. Hence, it is bounded by $C\left\|\mathcal{L}^{n} h\right\|_{t^{\prime}, q+t^{\prime}}^{-}$, which has already been estimated in the induction. For the remaining term (where no $f_{i}$ has been differentiated), we can replace $\varphi$ by $f_{1} \ldots f_{t} \cdot \varphi$ and assume that $\bar{v}_{i}=\partial_{\alpha(i)}$. In particular, $\bar{v}_{i}$ is well defined and $\mathcal{C}^{r+1}$ on a neighborhood of $\widetilde{W}$. 
Take some index $j$, we will estimate

$$
\int_{T^{n}\left(W_{j}\right)} \bar{v}_{1} \cdots \bar{v}_{t}\left(h_{n} \circ T^{-n}\right) \cdot \varphi \cdot \rho_{j} \circ T^{-n} .
$$

The decomposition of $\bar{v}_{i}$ as $w_{i}^{u}+w_{i}^{s}$ on a neighborhood of $T^{n}\left(W_{j}\right)$ is given by the following technical lemma.

LEMMA 6.5. Let $v$ be a vector field on a neighborhood of $\widetilde{W}$ with $|v|_{\mathcal{C}^{r+1}} \leq 1$. Then there exist $\mathcal{C}^{r+1}$ vector fields $w^{u}$ and $w^{s}$ on a neighborhood $U$ of $T^{n}\left(W_{j}\right)$, satisfying:

- $\quad$ for all $x \in T^{n}\left(W_{j}\right), w^{s}(x)$ is tangent to $T^{n}\left(W_{j}\right)$;

- $\quad\left|w^{s}\right|_{\mathcal{C}^{r+1}(U)} \leq C_{n}$ and $\left|w^{u}\right|_{\mathcal{C}^{r+1}(U)} \leq C_{n}$, where $C_{n}$ is a constant that may depend on $n$;

- $\quad\left|w^{s} \circ T^{n}\right| \mathcal{C}^{r}\left(W_{j}\right) \leq C$;

- $\left|D T^{n}(x)^{-1} w^{u}\left(T^{n} x\right)\right|_{\mathcal{C}^{p+q}\left(T^{-n} U\right)} \leq C \lambda^{-n}$.

The idea of the proof of the lemma is to decompose the tangent space at $y=T^{n}(x) \in$ $T^{n}\left(W_{j}\right)$ as the sum of the tangent space to $W$, and the image of the vertical direction $\{0\} \times \mathbb{R}^{d_{u}}$ under $D T^{n}(x)$. The decomposition $v=w^{u}+w^{s}$ is then obtained by projecting $v$ along these two directions. The estimates on $\left|w^{s} \circ T^{n}\right|$ and $\left|D T^{n}(x)^{-1} w^{u}\left(T^{n} x\right)\right|$ are then consequences of the smoothing properties of $T^{n}$ along $W_{j}$. This naive idea works well when $p+q \leq r-1$, but it yields only $C^{r}$ vector fields $w^{u}$ and $w^{s}$, which is not sufficient for our purposes if $r-1<p+q<r$. Hence, the rigorous proof of Lemma 6.5 involves additional regularization steps. As it is purely technical, it will be deferred to Appendix A.

As in Lemma 6.5, write $\bar{v}_{i}=w_{i}^{u}+w_{i}^{s}$. Then (6.4) is equal to

$$
\sum_{\sigma \in\{s, u\}^{t}} \int_{T^{n}\left(W_{j}\right)} w_{1}^{\sigma_{1}} \cdots w_{t}^{\sigma_{t}}\left(h_{n} \circ T^{-n}\right) \cdot \varphi \cdot \rho_{j} \circ T^{-n} .
$$

Take $\sigma \in\{s, u\}^{t}$, and let $k=\#\left\{i \mid \sigma_{i}=s\right\}$. Let $\pi$ be a permutation of $\{1, \ldots, t\}$ such that $\pi\{1, \ldots, k\}=\left\{i \mid \sigma_{i}=s\right\}$. Then

$$
\begin{aligned}
& \int_{T^{n}\left(W_{j}\right)} w_{1}^{\sigma_{1}} \cdots w_{t}^{\sigma_{t}}\left(h_{n} \circ T^{-n}\right) \cdot \varphi \cdot \rho_{j} \circ T^{-n} \\
& \quad=\int_{T^{n}\left(W_{j}\right)} \prod_{i=1}^{k} w_{\pi(i)}^{s} \prod_{i=k+1}^{t} w_{\pi(i)}^{u}\left(h_{n} \circ T^{-n}\right) \cdot \varphi \cdot \rho_{j} \circ T^{-n}+O\left(\|h\|_{p-1, q+1}\right) .
\end{aligned}
$$

Namely, the commutator of two $\mathcal{C}^{r+1}$ vector fields is a $\mathcal{C}^{r}$ vector field. Hence, if we exchange two vector fields, the difference is bounded by $C_{n}\|h\|_{p-1, q+1}$.

We integrate by parts with respect to the vector fields $w_{\pi(i)}^{s}$ : they are tangent to the manifold $W$, from where $\int_{W} w_{\pi(i)}^{s} f \cdot g=-\int_{W} f \cdot w_{\pi(i)}^{s} g+\int_{W} f g \cdot \operatorname{div} w_{\pi(i)}^{s}$. As $w_{\pi(i)}^{s}$ is $\mathcal{C}^{r+1}$ and the manifold $W$ is $\mathcal{C}^{\infty}$ with a $\mathcal{C}^{\infty}$ volume form (here, we use the fact that we work in a coordinate chart for which $\left.W \subset \mathbb{R}^{d_{s}} \times\{0\}\right)$, the divergence terms are bounded by $C_{n}\|h\|_{p-1, q+1}$. We get an integral $\int \prod_{k+1}^{t} w_{\pi(i)}^{u}\left(h_{n} \circ T^{-n}\right) \cdot \prod_{k}^{1} w_{\pi(i)}^{s}\left(\varphi \cdot \rho_{j} \circ T^{-n}\right)$. If we use one of the vector fields $w_{\pi(i)}^{s}$ to differentiate $\rho_{j} \circ T^{-n}$, then we obtain an integral 
of $t-k$ derivatives of $h$ against a function in $\mathcal{C}^{q+1+(t-k)}$, which is again bounded by $C_{n}\|h\|_{p-1, q+1}$. Hence,

$$
\begin{aligned}
\int_{T^{n}\left(W_{j}\right)} & \prod_{i=1}^{k} w_{\pi(i)}^{s} \prod_{i=k+1}^{t} w_{\pi(i)}^{u}\left(h_{n} \circ T^{-n}\right) \cdot \varphi \cdot \rho_{j} \circ T^{-n} \\
= & (-1)^{k} \int_{T^{n}\left(W_{j}\right)} \prod_{i=k+1}^{t} w_{\pi(i)}^{u}\left(h_{n} \circ T^{-n}\right) \cdot \prod_{i=k}^{1} w_{\pi(i)}^{s} \varphi \cdot \rho_{j} \circ T^{-n}+O\left(\|h\|_{p-1, q+1}\right) .
\end{aligned}
$$

Let $\bar{w}_{i}^{u}(x)=D T^{n}(x)^{-1} w_{i}^{u}\left(T^{n} x\right)$. This is a vector field on a neighborhood of $W_{j}$. Changing variables, the last integral in (6.5) is equal to

$$
\int_{W_{j}} \prod_{i=k+1}^{t} \bar{w}_{\pi(i)}^{u} h_{n} \cdot\left(\prod_{i=k}^{1} w_{\pi(i)}^{s} \varphi\right) \circ T^{n} \cdot \rho_{j} \cdot J_{W} T^{n}
$$

where $J_{W} T^{n}$ is the Jacobian of $T^{n}: W_{j} \rightarrow W$, as in the proof of (2.3).

We use the standard coordinate chart (of dimension $d_{s}$ ) on $W$, and write $w_{\pi(i)}^{s}=$ $\sum_{l=1}^{d_{s}} g_{\pi(i), l} \partial_{l}$ where $g_{\pi(i), l}$ is $\mathcal{C}^{r+1}$. Differentiating one of the functions $g_{\pi(i), l}$ yields another term bounded by $C_{n}\|h\|_{p-1, q+1}$. Consequently, up to $O\left(\|h\|_{p-1, q+1}\right)$, (6.6) is equal to the sum, for $l_{1}, \ldots, l_{k} \in\left\{1, \ldots, d_{s}\right\}^{k}$, of

$$
\int_{W_{j}} \prod_{i=k+1}^{t} \bar{w}_{\pi(i)}^{u} h_{n} \cdot\left(\prod_{i=1}^{k} \partial_{l_{i}} \varphi\right) \circ T^{n} \cdot \rho_{j} \cdot J_{W} T^{n} \cdot \prod_{i=1}^{k} g_{\pi(i), l_{i}} \circ T^{n} .
$$

Fix parameters $l_{1}, \ldots, l_{k}$. Let $F=\rho_{j} \cdot \prod_{i=1}^{k} g_{\pi(i), l_{i}} \circ T^{n}$. By Lemma 6.5, $|F|_{\mathcal{C}^{p+q}\left(W_{j}\right)} \leq C$. We want to estimate

$$
\int_{W_{j}} \prod_{i=k+1}^{t} \bar{w}_{\pi(i)}^{u} h_{n} \cdot\left(\prod_{i=1}^{k} \partial_{l_{i}} \varphi\right) \circ T^{n} \cdot J_{W} T^{n} \cdot F .
$$

Assume first that $t=p$ and $k=0$. The function $\varphi \circ T^{n}$ satisfies $\left|\varphi \circ T^{n}\right|_{\mathcal{C}^{p+q}\left(W_{j}\right)} \leq C$. As $|F|_{\mathcal{C}^{p+q}\left(W_{j}\right)} \leq C$, and the vector fields $w_{\pi(i)}^{u}$ have a $\mathcal{C}^{p+q}$ norm bounded by $\lambda^{-n}$ by Lemma 6.5, (3.5) (applied with $f=\left|\operatorname{det} D T^{n}\right|^{-1}$ ) implies that (6.7) is bounded by $\left.\left.C \lambda^{-p n}\|h\|_{p, q}|| \operatorname{det} D T^{n}\right|^{-1}\right|_{\mathcal{C}^{p+q}\left(W_{j}\right)} \cdot\left|J_{W} T^{n}\right|_{\mathcal{C}^{p+q}\left(W_{j}\right)}$.

In the other cases, $t-k<p$. It will be useful to smooth the test function. For $\varepsilon \leq \delta$ and $\bar{\varphi} \in \mathcal{C}_{0}^{q+t-k}(W, \mathbb{R})$, let $\mathbb{A}_{\varepsilon} \bar{\varphi} \in \mathcal{C}_{0}^{q+1+t-k}(\widetilde{W}, \mathbb{R})$ be obtained by convolving $\bar{\varphi}$ with a $\mathcal{C}^{\infty}$ mollifier whose support is of size $\varepsilon$. Let $a$ be the largest integer with $a<q+t-k$.

Lemma 6.6. For each $\bar{\varphi} \in \mathcal{C}^{q+t-k}$,

$$
\begin{aligned}
\left|\mathbb{A}_{\varepsilon} \bar{\varphi}\right|_{\mathcal{C}^{q+t-k}} & \leq C|\bar{\varphi}|_{\mathcal{C}^{q+t-k}} ; \\
\left|\mathbb{A}_{\varepsilon} \bar{\varphi}\right|_{\mathcal{C}^{q+1+t-k}} & \leq C \varepsilon^{-1}|\bar{\varphi}|_{\mathcal{C}^{q+t-k}} ; \\
\left|\mathbb{A}_{\varepsilon} \bar{\varphi}-\bar{\varphi}\right|_{\mathcal{C}^{a}} & \leq C \varepsilon^{q+t-k-a}|\bar{\varphi}|_{\mathcal{C}^{q+t-k}} .
\end{aligned}
$$

The proof of the above lemma is standard and is left to the reader. 
We apply this lemma to $\bar{\varphi}=\prod_{i=1}^{k} \partial_{l_{i}} \varphi$, with $\varepsilon=v^{(q+t-k) n /(q+t-k-a)}$. Then

$$
\left|\left(\mathbb{A}_{\varepsilon} \bar{\varphi}-\bar{\varphi}\right) \circ T^{n}\right|_{\mathcal{C}^{q+t-k}\left(W_{j}\right)} \leq C v^{(q+t-k) n} .
$$

Hence, by (3.5),

$$
\begin{aligned}
& \left|\int_{W_{j}} \prod_{i=k+1}^{t} \bar{w}_{\pi(i)}^{u} h_{n} \cdot\left(\prod_{i=1}^{k} \partial_{l_{i}} \varphi-\mathbb{A}_{\varepsilon} \prod_{i=1}^{k} \partial_{l_{i}} \varphi\right) \circ T^{n} \cdot J_{W} T^{n} \cdot F\right| \\
& \quad \leq\left.\left. C v^{(q+t-k) n} \lambda^{-(t-k) n}|| \operatorname{det} D T^{n}\right|^{-1}\right|_{\mathcal{C}^{q+t-k}\left(W_{j}\right)} \cdot\left|J_{W} T^{n}\right|_{\mathcal{C} q+t-k\left(W_{j}\right)}\|h\|_{p, q} .
\end{aligned}
$$

Note that, as $k \leq t, v^{(q+t-k) n} \lambda^{-(t-k) n} \leq v^{q n}$. Moreover, as $t-k<p$ and $\mathbb{A}_{\varepsilon} \bar{\varphi}$ is smoother than $\bar{\varphi}$,

$$
\int_{W_{j}} \prod_{i=k+1}^{t} \bar{w}_{\pi(i)}^{u} h_{n} \cdot\left(\mathbb{A}_{\varepsilon} \prod_{i=1}^{k} \partial_{l_{i}} \varphi\right) \circ T^{n} \cdot J_{W} T^{n} \cdot F=O\left(\|h\|_{p-1, q+1}\right) .
$$

To sum up, we have proved that

$$
\begin{aligned}
& \left|\int_{W} v_{1} \cdots v_{t}\left(\mathcal{L}^{n} h\right) \cdot \varphi\right| \leq O\left(\|h\|_{p-1, q+1}\right)+C \sum_{t^{\prime}<t}\left\|\mathcal{L}^{n} h\right\|_{t^{\prime}, q+t^{\prime}}^{-} \\
& \quad+\left(\sum_{j} \|\left.\left.\operatorname{det} D T^{n}\right|^{-1}\right|_{\mathcal{C}^{r}\left(W_{j}\right)} \cdot\left|J_{W} T^{n}\right|_{\mathcal{C}^{r}\left(W_{j}\right)}\right) \begin{cases}C\left(v^{q}\right)^{n}\|h\|_{p, q} & \text { if } t<p, \\
C \max \left(\lambda^{-p}, v^{q}\right)^{n}\|h\|_{p, q} & \text { if } t=p .\end{cases}
\end{aligned}
$$

By Lemma 6.2, the sum $\left.\left.\sum_{j}|| \operatorname{det} D T^{n}\right|^{-1}\right|_{\mathcal{C}^{r}\left(W_{j}\right)} \cdot\left|J_{W} T^{n}\right|_{\mathcal{C}^{r}\left(W_{j}\right)}$ is bounded independently of $n$. Together with the inductive assumption, this concludes the proof of Lemma 6.3.

We now prove (2.4) by induction over $p$. The case $p=0$ is given by (2.3).

Lemma 6.3 implies the inequality

$$
\left\|\mathcal{L}^{n} h\right\|_{p, q} \leq C \max \left(\lambda^{-p}, v^{q}\right)^{n}\|h\|_{p, q}+C_{n}\|h\|_{p-1, q+1} .
$$

To prove (6.8), we have only used the fact that $v$ is greater than the minimal contraction of $T$ in the stable direction, and $\lambda$ is less than the minimal expansion in the unstable direction. Let $\lambda^{\prime}>\lambda$ and $v^{\prime}<v$ satisfy the same conditions, we get in the same way

$$
\left\|\mathcal{L}^{n} h\right\|_{p, q} \leq C^{\prime} \max \left(\lambda^{\prime-p}, v^{\prime q}\right)^{n}\|h\|_{p, q}+C_{n}^{\prime}\|h\|_{p-1, q+1} .
$$

Finally, choose $n_{0}$ such that $C^{\prime} \max \left(\lambda^{\prime-p}, v^{\prime q}\right)^{n_{0}} \leq \max \left(\lambda^{-p}, v^{q}\right)^{n_{0}}$. Iterating (6.9) for $n=n_{0}$ (and remembering that $\left\|\mathcal{L}^{m} h\right\|_{p-1, q+1} \leq C\|h\|_{p-1, q+1}$ by the inductive assumption), we obtain (2.4).

\section{General perturbation results}

It is obvious from the previous discussion that all the results discussed so far-and in particular Lemmas 2.2 and 3.3-hold not only for the map $T$, but also for any map in a $\mathcal{C}^{r+1}$ open neighborhood $U$ of $T$, or for any composition of such maps. We will consider perturbations of $T$ as described in $\$ 2$, given by a probability measure $\mu$ on a space $\Omega$ and functions $g(\omega, \cdot) \in \mathcal{C}^{p+q}\left(X, \mathbb{R}_{+}\right)$, and we will assume that all the random diffeomorphisms $T_{\omega}$ we consider belong to the above set $U$. In this section, we will prove Theorem 2.7. 
LEMMA 7.1. There exists a constant $C>0$ such that, for any map $\widetilde{T} \in U$, for any $p \in \mathbb{N}^{*}$ and $q>0$ with $p+q<r$, for any $h \in \mathcal{B}^{p, q}$,

$$
\left\|\mathcal{L}_{T} h-\mathcal{L}_{\widetilde{T}} h\right\|_{p-1, q+1} \leq C d_{\mathcal{C}^{r+1}}(T, \widetilde{T})\|h\|_{p, q} .
$$

Proof. As usual, it is sufficient to prove it for $h \in \mathcal{C}^{r}$ by density. Let $\widetilde{W}$ be a full admissible leaf, given by an admissible graph $\chi \in \Xi_{i}$ defined on a ball $\bar{B}(x, A \delta)$. We will use Lemma 3.3 with $\gamma=\gamma_{0}>1$ : there exists a finite number of $\gamma$-admissible graphs $\chi_{1}, \ldots, \chi_{\ell}$, such that $\chi_{j}$ is defined on a ball $\bar{B}\left(x_{j}, A \gamma \delta\right) \subset\left(-2 r_{i(j)} / 3 \gamma, 2 r_{i(j)} / 3 \gamma\right)^{d_{s}}$ for some index $i(j)$, and such that the corresponding $\gamma$-admissible leaves cover $T^{-1}(W)$. Write $\rho_{j}$ for the corresponding partition of unity.

Take $\widetilde{T} \in U$. The projection on the first $d_{s}$ coordinates of

$$
\psi_{i(j)}^{-1} \circ \widetilde{T}^{-1} \circ T \circ \psi_{i(j)} \circ\left(\mathrm{Id}, \chi_{j}\right)\left(\bar{B}\left(x_{j}, \gamma A \delta\right)\right)
$$

contains the ball $\bar{B}\left(x_{j}, A \delta\right)$ if $U$ is small enough. Hence, it is possible to define a graph $\tilde{\chi}_{j}$ on $\bar{B}\left(x_{j}, A \delta\right)$ whose image is contained in $\psi_{i(j)}^{-1}\left(\widetilde{T}^{-1}(\widetilde{W})\right)$. Moreover, $\widetilde{T}^{-1}(W)$ is covered by the restrictions of these graphs to the balls $\bar{B}\left(x_{j}, \delta\right)$ if $U$ is small enough. Finally, $\left|\chi_{j}-\tilde{\chi}_{j}\right|_{\mathcal{C}^{p+q}\left(\bar{B}\left(x_{j}, A \delta\right)\right)} \leq C d_{\mathcal{C}^{r+1}}(T, \tilde{T})$.

Let $|\alpha| \leq p-1$, let $\varphi \in \mathcal{C}_{0}^{q+1+|\alpha|}(B(x, \delta), \mathbb{R})$, and set $\tilde{h}_{j}:=h \circ \psi_{i(j)}$. Then

$$
\int_{B(x, \delta)} \partial^{\alpha}\left(\left(\mathcal{L}_{T} h\right) \circ \psi_{i}\right)(\mathrm{Id}, \chi) \cdot \varphi=\sum_{|\beta| \leq|\alpha|} \sum_{j=1}^{\ell} \int_{B\left(x_{j}, \delta\right)} \partial^{\beta} \widetilde{h}_{j}\left(\mathrm{Id}, \chi_{j}\right) \cdot F_{\alpha, \beta, T, j} \cdot \rho_{j}
$$

for some functions $F_{\alpha, \beta, T, j}$ bounded in $\mathcal{C}^{q+1+|\beta|}$. The same equation holds for $\mathcal{L}_{\widetilde{T}} h$, with $\chi_{j}$ replaced by $\tilde{\chi}_{j}$ and $F_{\alpha, \beta, T, j}$ replaced by a function $F_{\alpha, \beta, \widetilde{T}, j}$ satisfying $\mid F_{\alpha, \beta, T, j}-$ $\left.F_{\alpha, \beta, \widetilde{T}, j}\right|_{\mathcal{C} q+|\beta|} \leq C d_{\mathcal{C}^{r+1}}(T, \widetilde{T})$.

For $1 \leq j \leq \ell$ and $|\beta| \leq|\alpha|$, we have

$$
\begin{aligned}
& \left|\int_{B\left(x_{j}, \delta\right)} \partial^{\beta} \widetilde{h}_{j}\left(\mathrm{Id}, \chi_{j}\right)\left(F_{\alpha, \beta, T, j}-F_{\alpha, \beta, \widetilde{T}, j}\right) \rho_{j}\right| \\
& \quad \leq C\|h\|_{p, q}\left|F_{\alpha, \beta, T, j}-F_{\alpha, \beta, \widetilde{T}, j}\right|_{\mathcal{C} q+|\beta|} \leq C\|h\|_{p, q} d_{\mathcal{C}^{r+1}}(T, \widetilde{T})
\end{aligned}
$$

and

$$
\begin{aligned}
& \left|\int_{B\left(x_{j}, \delta\right)} \partial^{\beta} \widetilde{h}_{j}\left(\mathrm{Id}, \chi_{j}\right) F_{\alpha, \beta, \widetilde{T}, j} \rho_{j}-\int_{B\left(x_{j}, \delta\right)} \partial^{\beta} \widetilde{h}_{j}\left(\mathrm{Id}, \tilde{\chi}_{j}\right) F_{\alpha, \beta, \widetilde{T}, j} \rho_{j}\right| \\
& \quad=\left|\int_{t=0}^{1} \int_{B\left(x_{j}, \delta\right)} D\left(\partial^{\beta} \widetilde{h}_{j}\right)\left(\mathrm{Id}, \tilde{\chi}_{j}+t\left(\chi_{j}-\tilde{\chi}_{j}\right)\right) \cdot\left(0, \chi_{j}-\tilde{\chi}_{j}\right) F_{\alpha, \beta, \widetilde{T}, j} \rho_{j}\right|
\end{aligned}
$$

When $t$ is fixed, each integral is an integral along an admissible graph, from where it is at most

$$
C\|h\|_{p, q}\left|\chi_{j}-\tilde{\chi}_{j}\right|_{\mathcal{C} q+|\beta|+1}\left|F_{\alpha, \beta, \widetilde{T}, j}\right|_{\mathcal{C}^{q+|\beta|+1}} \leq C\|h\|_{p, q} d_{\mathcal{C}^{r+1}}(T, \widetilde{T}) .
$$

Integrating over $t$, we get $(7.3) \leq C\|h\|_{p, q} d_{\mathcal{C}^{r+1}}(T, \widetilde{T})$. Combining this inequality with equations (7.2) and (7.1) yields the conclusion of the lemma. 
This lemma readily implies that, for any operator $\mathcal{L}_{\mu, g}$ satisfying the previous assumptions,

$$
\left\|\mathcal{L}_{\mu, g} h-\mathcal{L}_{T} h\right\|_{p-1, q+1} \leq C \Delta(\mu, g)\|h\|_{p, q},
$$

where $\Delta(\mu, g)$ is defined in (2.5).

When $g(\omega, x)=1$, Lemma 2.2 applied to compositions of operators of the form $\mathcal{L}_{T_{\omega}}$ immediately implies that

$$
\left\|\mathcal{L}_{\mu, g}^{n} h\right\|_{p, q} \leq C \max \left(\lambda^{-p}, v^{q}\right)^{n}\|h\|_{p, q}+C\|h\|_{p-1, q+1},
$$

which is sufficient to obtain spectral stability, by [13]. In particular, this suffices to prove Theorem 2.7 for deterministic perturbations.

However, in the general case, further arguments are required to obtain a uniform Lasota-Yorke-type inequality.

LEMMA 7.2. For any $M>1$ and any perturbation $(\mu, g)$ of $T$ as above, there exists a constant $C=C\left(M, \int|g(\omega, \cdot)|_{\mathcal{C}^{p+q}(X, \mathbb{R})} d \mu(\omega)\right)$ such that, for any $n \in \mathbb{N}$,

$$
\left\|\mathcal{L}_{\mu, g}^{n} h\right\|_{0, q} \leq C M^{n}\|h\|_{0, q}
$$

and

$$
\left\|\mathcal{L}_{\mu, g}^{n} h\right\|_{p, q} \leq C \max \left(\lambda^{-p}, \nu^{q}\right)^{n}\|h\|_{p, q}+C M^{n}\|h\|_{p-1, q+1} .
$$

Proof. We will prove (7.6), which is an extension to the perturbative case of (2.3), by adapting the proof of (2.3). The proof of (7.7) is similar, using the same ideas to extend the proof of (2.4).

The only problem comes from the functions $g\left(\omega_{i}, x\right)$, and a distortion argument will show that their contribution is small. Let $c=\int|g(\omega, \cdot)|_{\mathcal{C}^{q}} d \mu(\omega)$. Fix parameters $\bar{\omega}_{n}:=\left(\omega_{1}, \ldots, \omega_{n}\right) \in \Omega^{n}$. Fix also $\varepsilon>0$. Write $\tilde{g}_{i}(x)=g\left(\omega_{i}, x\right)+\varepsilon\left(\left|g\left(\omega_{i}, \cdot\right)\right|_{\mathcal{C}^{q}}\right) / c$.

We will write $T_{\bar{\omega}_{i}}=T_{\omega_{i}} \circ \ldots \circ T_{\omega_{1}}$. Let $W$ be an admissible leaf, $W_{1}, \ldots, W_{\ell}$ a covering of $T_{\bar{\omega}_{n}}^{-1} W$ by admissible leaves and $\rho_{1}, \ldots, \rho_{\ell}$ a corresponding partition of unity, as in the proof of (2.3). Let also $\varphi$ be a $\mathcal{C}^{q}$ test function. Then

$$
\begin{aligned}
& \int_{W}(\left.\prod_{i=1}^{n} g\left(\omega_{i}, T_{\omega_{i}}^{-1} \circ \cdots \circ T_{\omega_{n}}^{-1} x\right)\right) \mathcal{L}_{T_{\bar{\omega}_{n}}} h(x) \varphi(x) \\
& \quad=\sum_{j=1}^{\ell} \int_{W_{j}}\left|\operatorname{det} D T_{\bar{\omega}_{n}}(x)\right|^{-1} h(x)\left(\prod_{i=1}^{n} g\left(\omega_{i}, T_{\bar{\omega}_{i-1}} x\right)\right) \varphi \circ T_{\bar{\omega}_{n}}(x) J_{W} T_{\bar{\omega}_{n}}(x) \rho_{j}(x) .
\end{aligned}
$$

As $W_{j}$ is admissible, the last integral can be estimated using the $\mathcal{C}^{q}$ norm of $\prod_{i=1}^{n} g\left(\omega_{i}, T_{\bar{\omega}_{i-1}} x\right)$. As $\left|g\left(\omega_{i}, \cdot\right)\right|_{\mathcal{C}^{q}} \leq \frac{c}{\varepsilon} \widetilde{g}_{i}(x)$ by definition of $\widetilde{g}_{i}$, Lemma 6.1 shows that this norm is bounded by $C \exp (C c / \varepsilon) \prod_{i=1}^{n} \widetilde{g}_{i}\left(T_{\bar{\omega}_{i-1}} x\right)$ for any $x \in W_{j}$. Combining this estimate with the distortion arguments of the proof of (2.3), we obtain

$$
\begin{aligned}
& \left|\int_{W}\left(\prod_{i=1}^{n} g\left(\omega_{i}, T_{\omega_{i}}^{-1} \cdots T_{\omega_{n}}^{-1} x\right)\right) \mathcal{L}_{T_{\omega_{n}}} \cdots \mathcal{L}_{T_{\omega_{1}}} h(x) \varphi(x)\right| \\
& \quad \leq C \exp \left(C \frac{c}{\varepsilon}\right)\|h\|_{0, q} \int_{\widetilde{W}}\left|\operatorname{det} D T_{\bar{\omega}_{n}}\right|^{-1}\left(\prod_{i=1}^{n} \widetilde{g}_{i}\left(T_{\omega_{i}}^{-1} \cdots T_{\omega_{n}}^{-1} x\right)\right) .
\end{aligned}
$$


To estimate this last integral, consider the thickening $Z=\bigcup_{x \in \tilde{W}} W_{\rho}^{u}(x)$, where $W_{\rho}^{u}(x)$ is the local unstable manifold of $T_{\bar{\omega}_{n}}$ through $x$. Along this manifold, the function $\prod_{i=1}^{n} \tilde{g}_{i}\left(T_{\omega_{i}}^{-1} \cdots T_{\omega_{n}}^{-1} x\right)$ changes by a multiplicative factor of at most $C \exp (C c / \varepsilon)$, again by Lemma 6.1. Hence

$$
\begin{aligned}
& \int_{\widetilde{W}}\left|\operatorname{det} D T_{\bar{\omega}_{n}}\right|^{-1}\left(\prod_{i=1}^{n} \widetilde{g}_{i}\left(T_{\omega_{i}}^{-1} \cdots T_{\omega_{n}}^{-1} x\right)\right) \\
& \quad \leq C \exp \left(C \frac{c}{\varepsilon}\right) \rho^{-d_{u}} \int_{Z}\left|\operatorname{det} D T_{\bar{\omega}_{n}}\right|^{-1}\left(\prod_{i=1}^{n} \tilde{g}_{i}\left(T_{\omega_{i}}^{-1} \cdots T_{\omega_{n}}^{-1} x\right)\right) \\
& \quad \leq C \exp \left(C \frac{c}{\varepsilon}\right) \int_{T^{-n}(Z)} \prod_{i=1}^{n} \widetilde{g}_{i}\left(T_{\omega_{i-1}} \cdots T_{\omega_{1}} x\right) \\
& \leq C \exp \left(C \frac{c}{\varepsilon}\right) \int_{X} \prod_{i=1}^{n} \widetilde{g}_{i}\left(T_{\omega_{i-1}} \cdots T_{\omega_{1}} x\right) .
\end{aligned}
$$

Integrating over all possible values of $\bar{\omega}_{n}$, we finally obtain

$$
\begin{aligned}
\left\|\mathcal{L}_{\mu, g}^{n} h\right\|_{0, q} \leq & C\|h\|_{0, q} \exp \left(C \frac{c}{\varepsilon}\right) \\
& \times \int_{X} \int_{\Omega^{n}} \prod_{i=1}^{n}\left(g\left(\omega_{i}, T_{\omega_{i-1}} \cdots T_{\omega_{1}} x\right)+\varepsilon \frac{\left|g\left(\omega_{i}, \cdot\right)\right|_{\mathcal{C}^{q}}}{c}\right) d \mu\left(\omega_{1}\right) \cdots d \mu\left(\omega_{n}\right) .
\end{aligned}
$$

Integrating over $\omega_{n}$ gives a factor $1+\varepsilon$, as $\int g\left(\omega_{n}, y\right) d \mu\left(\omega_{n}\right)=1$ for any $y$. We can then proceed to integrate over $\omega_{n-1}, \omega_{n-2}, \ldots$, and get

$$
\left\|\mathcal{L}_{\mu, g}^{n} h\right\|_{0, q} \leq C\|h\|_{0, q} \exp \left(C \frac{c}{\varepsilon}\right)(1+\varepsilon)^{n} .
$$

The inequalities (7.4) and (7.7) are exactly what is needed to apply Theorem 1 in [13], which implies Theorem 2.7 .

\section{An abstract perturbation theorem}

Let $\mathcal{B}^{0} \supset \mathcal{B}^{1} \supset \cdots \supset \mathcal{B}^{s}, s \in \mathbb{N}$, be a finite family of Banach spaces, let $I \subset \mathbb{R}$ be a fixed open interval containing zero, and let $\left\{\mathcal{L}_{t}\right\}_{t \in I}$ be a family of operators acting on each of the above Banach spaces. Moreover, assume that

$$
\text { there exists } M>0 \text {, for all } t \in I, \quad\left\|\mathcal{L}_{t}^{n} f\right\|_{\mathcal{B}^{0}} \leq C M^{n}\|f\|_{\mathcal{B}^{0}}
$$

and

$$
\text { there exists } \alpha<M \text {, for all } t \in I, \quad\left\|\mathcal{L}_{t}^{n} f\right\|_{\mathcal{B}^{1}} \leq C \alpha^{n}\|f\|_{\mathcal{B}^{1}}+C M^{n}\|f\|_{\mathcal{B}^{0}} .
$$

Assume also that there exist operators $Q_{1}, \ldots, Q_{s-1}$ satisfying the following properties:

$$
\text { for all } j=1, \ldots, s-1 \text {, for all } i=j, \ldots, s, \quad\left\|Q_{j}\right\|_{\mathcal{B}^{i} \rightarrow \mathcal{B}^{i-j}} \leq C
$$

and, setting $\Delta_{0}(t):=\mathcal{L}_{t}$ and $\Delta_{j}(t):=\mathcal{L}_{t}-\mathcal{L}_{0}-\sum_{k=1}^{j-1} t^{k} Q_{k}$ for $j \geq 1$,

$$
\text { for all } t \in I \text {, for all } j=0, \ldots s \text {, for all } i=j, \ldots, s, \quad\left\|\Delta_{j}(t)\right\|_{\mathcal{B}^{i} \rightarrow \mathcal{B}^{i-j}} \leq C t^{j} .
$$


These assumptions mean that $t \mapsto \mathcal{L}_{t}$ is continuous at $t=0$ as a function from $\mathcal{B}^{i}$ to $\mathcal{B}^{i-1}$. In fact, $t \mapsto \mathcal{L}_{t}$ even has a Taylor expansion of order $s$, but the differentials take their values in weaker spaces. This setting can be applied to the case of smooth expanding maps (see [17] for the argument limited to the case $s=2$ ) and to the transfer operator associated to a perturbation of a smooth Anosov map as we will see in $\S 9$.

For $\varrho>\alpha$ and $\delta>0$, denote by $V_{\delta, \varrho}$ the set of complex numbers $z$ such that $|z| \geq \varrho$ and, for all $1 \leq k \leq s$, the distance from $z$ to the spectrum of $\mathcal{L}_{0}$ acting on $\mathcal{B}^{k}$ is $\geq \delta$.

THEOREM 8.1. Given a family of operators $\left\{\mathcal{L}_{t}\right\}_{t \in I}$ satisfying conditions (8.1)-(8.4) and setting

$$
R_{S}(t):=\sum_{k=0}^{s-1} t^{k} \sum_{\ell_{1}+\cdots+\ell_{j}=k}\left(z-\mathcal{L}_{0}\right)^{-1} Q_{\ell_{1}}\left(z-\mathcal{L}_{0}\right)^{-1} \cdots\left(z-\mathcal{L}_{0}\right)^{-1} Q_{\ell_{j}}\left(z-\mathcal{L}_{0}\right)^{-1}
$$

for all $z \in V_{\delta, \varrho}$ and $t$ small enough, we have that

$$
\left\|\left(z-\mathcal{L}_{t}\right)^{-1}-R_{S}(t)\right\|_{\mathcal{B}^{s} \rightarrow \mathcal{B}^{0}} \leq C|t|^{s-1+\eta}
$$

where $\eta=\log (\varrho / \alpha) / \log (M / \alpha)$.

Hence, the resolvent $\left(z-\mathcal{L}_{t}\right)^{-1}$ depends on $t$ in a $\mathcal{C}^{s-1+\eta}$ way at $t=0$, when viewed as an operator from $\mathcal{B}^{s}$ to $\mathcal{B}^{0}$.

Note that one of the results of [13] in the present setting reads

$$
\left\|\left(z-\mathcal{L}_{t}\right)^{-1}-\left(z-\mathcal{L}_{0}\right)^{-1}\right\|_{\mathcal{B}^{1} \rightarrow \mathcal{B}^{0}} \leq C|t|^{\eta} .
$$

Accordingly, one has Theorem 8.1 in the case $s=1$ where no assumption is made on the existence of the operators $Q_{j}$.

Proof of Theorem 8.1. Iterating the equation

$$
\left(z-\mathcal{L}_{t}\right)^{-1}=\left(z-\mathcal{L}_{0}\right)^{-1}+\left(z-\mathcal{L}_{t}\right)^{-1}\left(\mathcal{L}_{t}-\mathcal{L}_{0}\right)\left(z-\mathcal{L}_{0}\right)^{-1}
$$

and setting $A(z, t):=\left(\mathcal{L}_{t}-\mathcal{L}_{0}\right)\left(z-\mathcal{L}_{0}\right)^{-1}$, it follows that

$$
\begin{aligned}
\left(z-\mathcal{L}_{t}\right)^{-1} & =\sum_{j=0}^{s-2}\left(z-\mathcal{L}_{0}\right)^{-1} A(z, t)^{j}+\left(z-\mathcal{L}_{t}\right)^{-1} A(z, t)^{s-1} \\
& =\sum_{j=0}^{s-1}\left(z-\mathcal{L}_{0}\right)^{-1} A(z, t)^{j}+\left[\left(z-\mathcal{L}_{t}\right)^{-1}-\left(z-\mathcal{L}_{0}\right)^{-1}\right] A(z, t)^{s-1}
\end{aligned}
$$

Next, for each $j \in \mathbb{N}$ and $a \leq s$, using (8.4), we can write

$$
A(z, t)^{j}=\Delta_{a}(t)\left(z-\mathcal{L}_{0}\right)^{-1} A(z, t)^{j-1}+\sum_{\ell=1}^{a-1} t^{\ell} Q_{\ell}\left(z-\mathcal{L}_{0}\right)^{-1} A(z, t)^{j-1} .
$$


For $\epsilon=0$ or 1 , we can then prove by induction the formula, for all $1 \leq m \leq j$,

$$
\begin{aligned}
A(z, t)^{j}= & \sum_{k=1}^{m} \sum_{\substack{\ell_{1}+\cdots+\ell_{k-1}<s-\epsilon \\
\ell_{i}>0}} t^{\ell_{1}+\cdots+\ell_{k-1}} Q_{\ell_{1}}\left(z-\mathcal{L}_{0}\right)^{-1} \cdots \\
& \cdots Q_{\ell_{k-1}}\left(z-\mathcal{L}_{0}\right)^{-1} \Delta_{s-\epsilon-\ell_{1}-\cdots-\ell_{k-1}}(t)\left(z-\mathcal{L}_{0}\right)^{-1} A(z, t)^{j-k} \\
+ & \sum_{\substack{\ell_{1}+\cdots+\ell_{m}<s-\epsilon \\
\ell_{i}>0}} t^{\ell_{1}+\cdots+\ell_{m}} Q_{\ell_{1}}\left(z-\mathcal{L}_{0}\right)^{-1} \cdots Q_{\ell_{m}}\left(z-\mathcal{L}_{0}\right)^{-1} A(z, t)^{j-m} .
\end{aligned}
$$

In fact, for $m=1$ the above formula is just (8.7) for $a=s-\epsilon$. Next, suppose (8.8) is true for some $m$, then by (8.7) it follows that

$$
\begin{aligned}
& Q_{\ell_{1}}\left(z-\mathcal{L}_{0}\right)^{-1} \cdots Q_{\ell_{m}}\left(z-\mathcal{L}_{0}\right)^{-1} A(z, t)^{j-m}=Q_{\ell_{1}}\left(z-\mathcal{L}_{0}\right)^{-1} \cdots Q_{\ell_{m}}\left(z-\mathcal{L}_{0}\right)^{-1} \\
& \times\left[\Delta_{s-\epsilon-\sum_{i=1}^{m} \ell_{i}}(t)\left(z-\mathcal{L}_{0}\right)^{-1} A(z, t)^{j-m-1}\right. \\
& \left.\quad+\sum_{\ell_{m+1}=1}^{s-\epsilon-\sum_{i=1}^{m} \ell_{i}-1} t^{\ell_{m+1}} Q_{\ell_{m+1}}\left(z-\mathcal{L}_{0}\right)^{-1} A(z, t)^{j-m-1}\right] .
\end{aligned}
$$

Substituting the above formula in (8.8) we have the formula for $m+1$.

We can now easily estimate the terms in which a $\Delta_{i}$ appears. In fact, $\|A(z, t)\|_{\mathcal{B}^{s}} \leq C$, and (8.3) and (8.4) readily imply that

$\left\|Q_{\ell_{1}}\left(z-\mathcal{L}_{0}\right)^{-1} \cdots Q_{\ell_{k-1}}\left(z-\mathcal{L}_{0}\right)^{-1} \Delta_{s-\epsilon-\sum_{j=1}^{k-1} \ell_{j}}(t)\left(z-\mathcal{L}_{0}\right)^{-1}\right\|_{\mathcal{B}^{s} \rightarrow \mathcal{B}^{\epsilon}} \leq C|t|^{s-\epsilon-\sum_{j=1}^{k-1} \ell_{j}}$.

The theorem follows then from (8.6), using (8.8) with $\epsilon=0$ and $m=j$ to estimate the terms $\left(z-\mathcal{L}_{0}\right)^{-1} A(z, t)^{j}$, and (8.8) with $\epsilon=1$ and $m=j=s-1$ together with (8.5) to show that $\left\|\left[\left(z-\mathcal{L}_{t}\right)^{-1}-\left(z-\mathcal{L}_{0}\right)^{-1}\right] A(z, t)^{s-1}\right\|_{\mathcal{B}^{s} \rightarrow \mathcal{B}^{0}} \leq C|t|^{s-1+\eta}$.

\section{Differentiability results}

In this section, we prove Theorem 2.8 by applying Theorem 8.1 . To simplify the exposition, we will abuse notation and systematically ignore the coordinate charts of the manifold $X$. As we have carefully discussed in the previous sections, this does not create any problem.

To start with, let us assume that $t_{*}$ is so small that $\left\{T_{t}: t \in\left[-t_{*}, t_{*}\right]\right\}$ is contained in the neighborhood $U$ of $T_{0}$ in which the estimates of the Lasota-Yorke inequality hold uniformly.

By Taylor's formula we have, for each $f \in \mathcal{C}^{r}$ and $s \leq r$,

$$
\mathcal{L}_{T_{t}} f=\left.\sum_{k=0}^{s-1} \frac{1}{k !} \frac{d^{k}}{d t^{k}} \mathcal{L}_{T_{t}} f\right|_{t=0}+\int_{0}^{t} d t_{1} \cdots \int_{0}^{t_{s-1}} d t_{s}\left(\frac{d^{s}}{d t^{s}} \mathcal{L}_{t} f\right)\left(t_{s}\right) .
$$

Next, for $1 \leq k \leq s-1$,

$$
\left.\frac{d^{k}}{d t^{k}} \mathcal{L}_{T_{t}} f(x)\right|_{t=0}=\left.\sum_{\ell=1}^{k} \sum_{|\alpha|=\ell} J_{\alpha}(k, t, x)\left(\mathcal{L}_{T_{t}} \partial^{\alpha} f\right)(x)\right|_{t=0}=: k ! Q_{k} f(x)
$$

for appropriate functions $J_{\alpha}(k, t, \cdot) \in \mathcal{C}^{r}(X, \mathbb{R})$. 
We are now ready to check the applicability of Theorem 8.1. First, let us define $\mathcal{B}^{i}:=\mathcal{B}^{p-1+i, q+s-i}$. Conditions (8.1) and (8.2) hold with $\alpha=\max \left(\lambda^{-p}, \nu^{q+s-1}\right)$ by our choice of $t_{*}$, and $M=1$ by (7.5). Moreover, for $1 \leq i \leq s$, the essential spectrum of $\mathcal{L}$ acting on $\mathcal{B}^{i}$ is contained in $\left\{|z| \leq \max \left(\lambda^{-(p-1+i)}, v^{q+s-i}\right)\right\}$. Hence, for $1 \leq i \leq s$, $\operatorname{sp}\left(\mathcal{L}: \mathcal{B}^{i} \rightarrow \mathcal{B}^{i}\right) \cap\left\{|z|>\max \left(\lambda^{-p}, \nu^{q}\right)\right\}$ is composed of isolated eigenvalues of finite multiplicity. In particular, $V_{\delta, \varrho}$ is discrete.

From the definition of the norms it follows straightforwardly that, for each multi-index $\alpha$ with $|\alpha|=j, \partial^{\alpha}$ is a bounded operator from $\mathcal{B}^{p, q}$ to $\mathcal{B}^{p-j, q+j}$. From this, Condition (8.3) readily follows. By (9.1) and (9.2), it follows that $\Delta_{k}$ is given by the last term in (9.1). By the previous arguments

$$
\left\|\frac{d^{k}}{d t^{k}} \mathcal{L}_{T_{t}}(f)\right\|_{p-k, q+k} \leq C\|f\|_{p, q},
$$

which obviously implies Condition (8.4).

\section{A. Appendix. Distortion estimates}

In this appendix, we prove Lemma 6.5. Recall that $\widetilde{W}_{j}$ and $\widetilde{W}$ are considered as subsets of $\mathbb{R}^{d_{s}} \times\{0\} \subset \mathbb{R}^{d}$. For $y \in \widetilde{W}$, let $F(y)=\mathbb{R}^{d_{s}} \times\{0\}$. This defines a $\mathcal{C}^{\infty}$ field of planes of dimension $d_{s}$ on $\widetilde{W}$. For $x \in \widetilde{W}_{j}$, set also $E(x)=D T^{n}(x)\left(\{0\} \times \mathbb{R}^{d_{u}}\right)$. Let $\vartheta_{\varepsilon}$ be a $\mathcal{C}^{\infty}$ mollifier of size $\varepsilon$ on $\mathbb{R}^{d_{s}}$. Define a new field of planes of dimension $d_{u}$ on $T^{n}\left(W_{j}\right)$ by $E_{\varepsilon}(y)=\int E\left(T^{-n} y+z\right) \vartheta_{\varepsilon}(z) d z$. It is still uniformly transversal to $F$, and it is $\mathcal{C}^{r+1}$ along $T^{n}\left(W_{j}\right)$, even though $E$ was only $\mathcal{C}^{r}$, thanks to the regularizing effect of $\vartheta_{\varepsilon}$. Note that a convolution usually shrinks slightly the domains of definition of functions. However, at the beginning, our functions are defined on larger sets $\widetilde{W}$ and $\widetilde{W}_{j}$. Hence, we can safely forget about this issue in what follows.

A vector field $v$ with $|v|_{\mathcal{C}^{r+1}} \leq 1$ can be decomposed along $T^{n}\left(W_{j}\right)$ as $v=w^{u}+w^{s}$ where $w^{u}(y) \in E_{\varepsilon}(y)$ and $w^{s}(y) \in F(y)$. We will first estimate the norms of this decomposition along $W$, and prove that, if $\varepsilon$ is small enough,

$$
\left|w^{s} \circ T^{n}\right|_{\mathcal{C}^{r}\left(W_{j}\right)} \leq C
$$

and

$$
\left|D T^{n}(x)^{-1} w^{u}\left(T^{n} x\right)\right|_{\mathcal{C}^{p+q}\left(W_{j}\right)} \leq C \lambda^{-n} .
$$

Then, the second step of the proof will be to extend this decomposition to a neighborhood of $T^{n}\left(W_{j}\right)$ so that the conclusions of Lemma 6.5 hold.

We will first estimate the $\mathcal{C}^{r}$ norm of $x \mapsto E(x)$ along $W_{j}$. If

$$
D T^{n}(x)=\left(\begin{array}{cc}
A^{n}(x) & B^{n}(x) \\
0 & D^{n}(x)
\end{array}\right),
$$

the projection on $E(x)$ is given by

$$
\left(\begin{array}{ll}
0 & U^{n} \\
0 & \text { Id }
\end{array}\right)
$$

where $U^{n}(x)=B^{n}(x) D^{n}(x)^{-1}$. Let $x_{0}$ be an arbitrary point of $W_{j}$, we will work on a small neighborhood of $x_{0}$. For $k=1, \ldots, n-1$, let $\theta_{k}$ be a chart on a neighborhood of 
$T^{k} x_{0}$ such that $\theta_{k}\left(T^{k}\left(W_{j}\right)\right) \subset \mathbb{R}^{d_{s}} \times\{0\}$ and $D \theta_{k}\left(T^{k} x_{0}\right) D T^{k}\left(\{0\} \times \mathbb{R}^{d_{u}}\right)=\{0\} \times \mathbb{R}^{d_{u}}$. As the manifolds $T^{k}\left(W_{j}\right)$ are uniformly $\mathcal{C}^{r+1}$ (locally, they are admissible leaves) and uniformly transversal to $D T^{k}\left(\{0\} \times \mathbb{R}^{d_{u}}\right)$, we can choose such charts with a uniformly bounded $\mathcal{C}^{r+1}$ norm. Set also $\theta_{0}=\operatorname{Id}$ and $\theta_{n}=\operatorname{Id}$ (this is coherent with the previous choices as $W_{j}$ and $W$ are already assumed to be subsets of $\mathbb{R}^{d_{s}} \times\{0\}$ ).

Let $\widetilde{T}_{k}=\theta_{k} \circ T \circ \theta_{k-1}^{-1}$, and $\widetilde{T}^{k}=\widetilde{T}_{k} \circ \cdots \circ \widetilde{T}_{1}$. For $y \in \mathbb{R}^{d_{s}} \times\{0\}$, we can write

$$
D \widetilde{T}_{k}(y)=\left(\begin{array}{cc}
A_{k}(y) & B_{k}(y) \\
0 & D_{k}(y)
\end{array}\right),
$$

with $\left|A_{k}(y)\right| \leq v,\left|D_{k}(y)^{-1}\right| \leq \lambda$. For $0 \leq k \leq n-2$, as $B_{k}\left(\widetilde{T}^{k} x_{0}\right)=0$, we can reduce the neighborhood of $x_{0}$ and assume that $\left|B_{k}(y)\right| \leq v^{k}$. For $x \in \mathbb{R}^{d_{s}} \times\{0\}$, let

$$
D \widetilde{T}^{k}(x)=\left(\begin{array}{cc}
A^{k}(x) & B^{k}(x) \\
0 & D^{k}(x)
\end{array}\right) .
$$

If $U^{k}(x)=B^{k}(x) D^{k}(x)^{-1}$, we can write

$$
\begin{gathered}
D^{k+1}(x)^{-1}=D^{k}(x)^{-1} D_{k+1}\left(\widetilde{T}^{k} x\right)^{-1}, \\
U^{k+1}(x)=\left(A_{k+1}\left(\widetilde{T}^{k} x\right) U^{k}(x)+B_{k+1}\left(\widetilde{T}^{k} x\right)\right) D_{k+1}\left(\widetilde{T}^{k} x\right)^{-1} .
\end{gathered}
$$

We have $\left|D_{k+1}\left(\widetilde{T}^{k} x\right)^{-1}\right| \leq \lambda^{-1}$, and its derivatives with respect to $x$ are bounded by $C \nu^{-k}$ by uniform contraction of $\widetilde{T}$ along $\mathbb{R}^{d_{s}} \times\{0\}$. Hence,

$$
\left|D^{k}(x)^{-1}\right| \mathcal{C}^{r} \leq \prod_{\ell=1}^{k}\left(\lambda^{-1}+C v^{-\ell}\right) \leq C \lambda^{-k} .
$$

In the same way, as $\left|B_{k}(y)\right| \leq v^{k}$ for $k \leq n-2$ by the smallness of the neighborhood of $x_{0}$,

$$
\left|U^{k+1}\right| \mathcal{C}^{r} \leq\left(\left(\nu+C v^{k}\right)\left|U^{k}\right| \mathcal{C}^{r}+C \nu^{k}\right)\left(\lambda^{-1}+C \nu^{k}\right) .
$$

This implies $\left|U^{n-1}\right| \mathcal{C}^{r} \leq C \nu^{n}$, from where

$$
\left|U^{n}(x)\right|_{\mathcal{C}^{r}} \leq C .
$$

Let $v$ be a $\mathcal{C}^{r+1}$ vector field on a neighborhood of $T^{n}\left(W_{j}\right)$. For $x \in W_{j}$ and $y=T^{n}(x)$, write $v(y)=\left(v_{1}(y), v_{2}(y)\right)$ the decomposition of $v$ along $\mathbb{R}^{d_{s}} \times \mathbb{R}^{d_{u}}$. Then the decomposition of $v(y)$ in $w^{u}(y)+w^{s}(y)$ is given by

$$
\begin{gathered}
w^{u}(y)=\left(\left[\int U^{n}(x+z) \vartheta_{\varepsilon}(z) d z\right] v_{2}(y), v_{2}(y)\right), \\
w^{s}(y)=\left(v_{1}(y)-\left[\int U^{n}(x+z) \vartheta_{\varepsilon}(z) d z\right] v_{2}(y), 0\right) .
\end{gathered}
$$

Namely, these vectors satisfy $w^{u}+w^{s}=v, w^{u}$ is tangent to $E_{\varepsilon}(y)$ and $w^{s}$ is tangent to $\mathbb{R}^{d_{s}} \times\{0\}$.

As the $\mathcal{C}^{r}$ norm of $U^{n}$ is bounded, by (A.4), this proves (A.1). Moreover,

$$
\begin{aligned}
& D T^{n}(x)^{-1} w^{u}\left(T^{n} x\right) \\
& \quad=\left(A^{n}(x)^{-1}\left[\int\left(U^{n}(x+z)-U^{n}(x)\right) \vartheta_{\varepsilon}(z) d z\right] v_{2}\left(T^{n} x\right), D^{n}(x)^{-1} v_{2}\left(T^{n} x\right)\right) .
\end{aligned}
$$


Hence, (A.3) implies that the $\mathcal{C}^{r}$ norm of the second component is bounded by $C \lambda^{-n}$. On the other hand, the first component is not necessarily small in the $\mathcal{C}^{r}$ topology. However, as $p+q<r$, its $\mathcal{C}^{p+q}$ norm is bounded by

$$
C\left|A_{n}(x)^{-1}\right|_{\mathcal{C}^{p+q}\left(W_{j}\right)} \varepsilon^{r-(p+q)},
$$

which can be made arbitrarily small by choosing $\varepsilon$ small enough. This proves (A.2).

We still have to extend $w^{s}$ and $w^{u}$ to a neighborhood of $T^{n}\left(W_{j}\right)$. Let $\pi: \mathbb{R}^{d} \rightarrow \mathbb{R}^{d_{s}}$ be the projection on the first $d_{s}$ components. A naive idea to construct an extension $w_{1}^{u}$ of $w^{u}$ is to set

$$
w_{1}^{u}(y)=D T^{n}\left(T^{-n} y\right) D T^{n}\left(\pi T^{-n} y\right)^{-1} w^{u}\left(T^{n} \pi T^{-n} y\right)
$$

In other words, we extend $w^{u}$ so that the vector field $D T^{n}(x)^{-1} w_{1}^{u}\left(T^{n} x\right)$ is constant along the vertical planes $\{\eta\} \times \mathbb{R}^{d_{u}}$. By (A.2), this extension satisfies

$$
\left|D T^{n}(x)^{-1} w_{1}^{u}\left(T^{n} x\right)\right|_{\mathcal{C}^{p+q}(V)} \leq C \lambda^{-n}
$$

for some neighborhood $V$ of $W_{j}$. The vector field $w_{1}^{u}$ is unfortunately only $\mathcal{C}^{r}$, which means that we will have to regularize it.

Lemma A.1. Let $G: \mathbb{R}^{d} \rightarrow \mathbb{R}$ be a $\mathcal{C}^{r}$ function whose restriction to $\mathbb{R}^{d_{s}} \times\{0\}$ is $\mathcal{C}^{r+1}$. Then, for every $\varepsilon>0$, there exists a $\mathcal{C}^{r+1}$ function $H: \mathbb{R}^{d} \rightarrow \mathbb{R}$ such that:

(1) $|H|_{\mathcal{C}^{r+1}} \leq C_{\varepsilon}|G|_{\mathcal{C}^{r}}+C|G|_{\mathcal{C}^{r+1}\left(\mathbb{R}^{d_{s}} \times\{0\}\right)}$;

(2) The restrictions of $G$ and $H$ to $\mathbb{R}^{d_{s}} \times\{0\}$ are equal;

(3) $|G-H|_{\mathcal{C}^{p+q}} \leq \varepsilon|G|_{\mathcal{C}^{r}}$.

Proof. Replacing $G$ and $H$ by $G-G \circ \pi$ and $H-G \circ \pi$, we can assume without loss of generality that $G=0$ on $\mathbb{R}^{d_{s}} \times\{0\}$. Let $H_{0}$ be obtained by convolving $G$ with a $\mathcal{C}^{\infty}$ mollifier $\vartheta_{\varepsilon}$ of size $\varepsilon$ in $\mathbb{R}^{d}$. Let finally $H=H_{0}-H_{0} \circ \pi$. The first and second conclusions of the lemma are clearly satisfied by $H$.

The functions $G$ and $H_{0}$ satisfy $\left|G-H_{0}\right|_{\mathcal{C}^{p+q}} \leq C \varepsilon^{r-(p+q)}|G|_{\mathcal{C}^{r}}$, which can be made arbitrarily small. To conclude, we have to prove that the $\mathcal{C}^{p+q}$ norm of $H_{0} \circ \pi$ is arbitrarily small. For $\eta \in \mathbb{R}^{d_{s}}$, we have

$$
H_{0}(\eta, 0)=\int G\left(\eta+\eta^{\prime}, \xi^{\prime}\right) \vartheta_{\varepsilon}\left(\eta^{\prime}, \xi^{\prime}\right) d \eta^{\prime} d \xi^{\prime}
$$

As $G=0$ on $\mathbb{R}^{d_{s}} \times\{0\}$, the $\mathcal{C}^{p+q}$ norm of the restriction of $G$ to $\mathbb{R}^{d_{s}} \times\{\xi\}$ is bounded by $C|\xi|^{r-(p+q)}|G|_{\mathcal{C}^{r}}$. Together with (A.6), this implies $\left|H_{0} \circ \pi\right|_{\mathcal{C}^{p+q}} \leq C \varepsilon^{r-(p+q)}|G|_{\mathcal{C}^{r}}$.

Applying this lemma to the components of $w_{1}^{u}$, we obtain a new vector field $w_{2}^{u}$, which coincides with $w^{u}$ on $T^{n}\left(W_{j}\right)$, belongs to $\mathcal{C}^{r+1}$, and satisfies $\left|w_{1}^{u}-w_{2}^{u}\right|_{\mathcal{C}^{p+q}} \leq \varepsilon$. Choosing $\varepsilon$ small enough, this together with (A.5) implies

$$
\left|D T^{n}(x)^{-1} w_{2}^{u}\left(T^{n} x\right)\right|_{\mathcal{C}^{p+q}(V)} \leq C \lambda^{-n}
$$

Let finally $w^{s}=v-w_{2}^{u}$, the vector fields $w^{s}$ and $w_{2}^{u}$ satisfy all the conclusions of Lemma 6.5 . 
Acknowledgements. We wish to thank V. Baladi, G. Keller and D. Ruelle for their helpful discussions. Last, but most of all, it is a pleasure to acknowledge our debt to D. Dolgopyat. In fact, a couple of years ago, while in Rome, he told the second named author that one should 'project along the unstable direction' and a few other words. It is a fact that the above sentence is the key idea of the present work. It is only to our detriment that it took us so long to figure out the meaning and to understand how to implement it. C. Liverani acknowledges the support of M.I.U.R. and the hospitality of Paris VII and the Georgia Institute of Technology where part of the paper was written.

\section{REFERENCES}

[1] D. V. Anosov. Geodesic flows on closed Riemannian manifolds of negative curvature. Proc. Steklov Inst. Math. 90 (1967), 1-209.

[2] D. V. Anosov and Ya. G. Sinai. Certain smooth ergodic systems. (Russian) Usp. Mat. Nauk 22(5) (1967), 107-172 (Engl. Transl. Russian Math. Surveys 22(5) (1967), 103-167).

[3] V. I. Bakhtin. A direct method for constructing an invariant measure on a hyperbolic attractor. (Russian) Izv. Ross. Akad. Nauk Ser. Mat. (56) (1992), 934-957 (Engl. Transl. Russian Acad. Sci. Izv. Math. 41(2) (1993), 207-227).

[4] V. Baladi. Positive Transfer Operators and Decay of Correlations (Advanced Series in Nonlinear Dynamics, 16). World Scientific, 2000.

[5] V. Baladi. Anisotropic Sobolev spaces and dynamical transfer operators: $\mathcal{C}^{\infty}$ foliations. Preprint.

[6] V. Baladi and M. Tsujii. Anisotropic Hölder and Sobolev spaces for hyperbolic diffeomorphisms. Preprint.

[7] M. Blank, G. Keller and C. Liverani. Ruelle-Perron-Frobenius spectrum for Anosov maps. Nonlinearity 15(6) (2001), 1905-1973.

[8] R. Bowen. Equilibrium States and the Ergodic Theory of Anosov Diffeomorphisms (Lecture Notes in Mathematics, 470). Springer, Berlin, New York, 1975.

[9] D. Dolgopyat. On differentiability of SRB states for partially hyperbolic systems. Invent. Math. 155(2) (2004), 389-449.

[10] D. Fried. Meromorphic zeta functions for analytic flows. Comm. Math. Phys. 174(1) (1995), 161-190.

[11] L. Hörmander. The Analysis of Linear Partial Differential Operators I (Grundlehren der Mathematischen Wissenschaften, 256). Springer, 1990.

[12] G. Keller, On the rate of convergence to equilibrium in one-dimensional systems. Comm. Math. Phys. 96(2) (1984), 181-193.

[13] G. Keller and C. Liverani. Stability of the spectrum for transfer operators. Ann. Scuola Norm. Sup. Pisa (4) 28 (1999), 141-152.

[14] A. Yu. Kitaev. Fredholm determinants for hyperbolic diffeomorphisms of finite smoothness. Nonlinearity 12(1) (1999), 141-179.

[15] A. Lasota and J. A. Yorke. On the existence of invariant measures for piecewise monotonic transformations. Trans. Amer. Math. Soc. 186 (1963), 481-488.

[16] C. Liverani. Decay of correlations. Ann. of Math. 142 (1995), 239-301.

[17] C. Liverani. Invariant measures and their properties. A functional analytic point of view. Dynamical Systems. Part II: Topological Geometrical and Ergodic Properties of Dynamics. Pubblicazioni della Classe di Scienze, Scuola Normale Superiore, Pisa. Centro di Ricerca Matematica 'Ennio De Giorgi': Proceedings. Scuola Normale Superiore, Pisa, 2004.

[18] C. Liverani. Rigorous numerical investigation of the statistical properties of piecewise expanding mapsA feasibility study. Nonlinearity 14 (2001), 463-490.

[19] C. Liverani. On contact Anosov flows. Ann. of Math. 159 (2004), 1275-1312.

[20] G. A. Margulis. Certain measures that are connected with U-flows on compact manifolds. Funct. Anal. Appl. 4 (1970), 55-67.

[21] J. N. Mather. Characterization of Anosov diffeomorphisms. Indag. Math. 30 (1968), 479-483. 
[22] Ya. B. Pesin and Ya. G. Sinai. Gibbs measures for partially hyperbolic attractors. Ergod. Th. \& Dynam. Sys. 2(3-4) (1982), 417-438.

[23] M. Pollicott. Stability of mixing rates for Axiom A attractors. Nonlinearity 16 (2003), 567-578.

[24] D. Ruelle. A measure associated with axiom-A attractors. Amer. J. Math. 98 (1976), 619-654.

[25] D. Ruelle. The thermodynamics formalism for expanding maps. Comm. Math. Phys. 125 (1989), 239-262.

[26] D. Ruelle. Differentiation of SRB states. Comm. Math. Phys. 187 (1997), 227-241.

[27] D. Ruelle, Correction and complements: 'Differentiation of SRB states' [Comm. Math. Phys. 187 (1997), no. 1, 227-241]. Comm. Math. Phys. 234(1) (2003), 185-190.

[28] H. H. Rugh. The correlation spectrum for hyperbolic analytic maps. Nonlinearity 5(6) (1992), 1237-1263.

[29] H. H. Rugh. Fredholm determinants for real-analytic hyperbolic diffeomorphisms of surfaces. XIth International Congress of Mathematical Physics (Paris, 1994). International Press, Cambridge, MA, 1995, 297-303.

[30] H. H. Rugh. Generalized Fredholm determinants and Selberg zeta functions for Axiom A dynamical systems. Ergod. Th. \& Dynam. Sys. 16(4) (1996), 805-819.

[31] Ya. G. Sinai. Gibbs measures in ergodic theory. Usp. Mat. Nauk 27(4) (1972), 21-64 (Engl. Transl. Russian Math. Surveys 27(4) (1972), 21-70). 